\title{
1 Combined Non-Destructive Testing (NDT) method for the evaluation of the 2 mechanical characteristics of Ultra High Performance Fibre Reinforced 3 Concrete (UHPFRC)
}

4 Ourania Tsioulou, Andreas Lampropoulos* and Spyridon Paschalis

5 School of Environment and Technology, University of Brighton, Lewes Road, Brighton, BN2 4GJ,

6 UK

$7 \quad *$ Corresponding author, email: a.lampropoulos@ brighton.ac.uk

8

9

\begin{abstract}
Ultra-High Performance Fibre Reinforced Concrete is a material which is becoming increasingly popular in structural applications, mainly due to its superior mechanical characteristics. The mechanical properties of this material are of high importance and the development of non-destructive techniques is vital for the evaluation of the mechanical characteristics of existing structures. In the current study, Ultra-High Performance Fibre Reinforced Concrete with different amounts of steel fibres has been examined. Compressive and tensile tests have been conducted alongside with Ultrasonic Pulse Velocity and Rebound Hammer measurements and the development of appropriate empirical non-destructive models has been examined.
\end{abstract}

Keywords: UHPFRC, NDT, Rebound Hammer, Ultrasonic, SonReb,

\section{Introduction}

Ultra High Performance Fibre Reinforced Concrete (UHPFRC) is a novel material with superior strength and energy absorption [1]. UHPFRC composition differs to that of an ordinary concrete as it contains low water over cement ratio, silica fume, steel fibres and silica sand. Steel fibres content is one of the most crucial parameters of the mix and important mechanical characteristics such as the tensile strength, the flexural strength and the ductility of UHPFRC elements are highly affected by the percentage of steel fibres. According to previously published studies [2-7], increment of steel fibres amount results to an increment of the flexural strength. In literature [1, 8-11], there are several investigations on the mechanical properties of UHPFRC based on conventional destructive methods. However, there are very limited studies on the evaluation of the mechanical properties of UHPFRC using Non-Destructive Testing (NDT) [12-13] and there are not any published studies to date on combined NDT methods for the estimation of the mechanical characteristics of UHPFRC. Washer $e t$ al. [12] investigated the applicability of Ultrasonic Pulse Velocity (UPV) on UHPFRC, and the effect of steel fibres content on the wave velocity. The effectiveness of UPV was also examined by Hassan and Jones [13] and the need for further investigation was highlighted. 
NDT methods are useful for the evaluation of the condition of structures, by performing indirect assessment of concrete properties. NDT has many advantages as structural damage during testing is reduced, is relatively simple and less time consuming, and there is possibility of taking measurements even from structures where cores cannot be drilled [14-15]. NDT methods have also been proposed for the assessment of the damage and for the survey of detailed condition of concrete structures and road pavements [16]. There are several NDT methods and two of the most commonly used for in-situ applications are the Rebound Hammer (RH) and the UPV techniques. RH test is a quick method for determining the quality of concrete based on its surface hardness [17]. Schmidt rebound hammer is used to measure the hardness of the surface. Schmidt hammer consists of a mass and a spring which is sliding along a bar which impacts on the end of a steel plunger. After the impact on the concrete surface, the mass rebounds from the steel plunger and moves an index rider. Schmidt hammers normally measure either R-value or Q-value. R-value is the rebound index which is being calculated by the ratio of paths of the mass before and after impact. Q-value or coefficient of restitution is the ratio of kinetic energies of the mass before and after the impact. The energy absorbed by the concrete depends on the stress-strain characteristics of concrete and hence on the modulus of Elasticity and the maximum compressive strength. UPV method [18-19] is based on measurements of the velocity of an ultrasonic pulse which is generated by an electro-acoustical transducer through concrete. Using UPV results, the structure of concrete alongside with its density and any cracks or defects can be evaluated. In the literature, there are various proposed models for conventional concrete for the correlation of RH index values and UPV with concrete compressive strength and modulus of Elasticity [26, 38-39]. In the last few years, combination of more than one method is becoming more popular since they can offer improved reliability and limited errors compared to respective results of individual methods [21, 40-44]. SonReb method is one of them. The term SonReb is created by combing the terms 'Sonic' and 'Rebound' and is a method which is based on the combination of UPV and RH tests results; and until now has only been used for the development of models appropriate for the prediction of conventional concrete compressive strength. This combined method is more accurate than the single NDT methods as takes into consideration two parameters (UPV and RH) which are influenced in different ways by similar factors related to concrete density and hardness. The SonReb method is an empirical method to determine appropriate models for the correlation of the mechanical characteristics (normally compressive strength) with the UPV and RH index values. By using multiple-regression analysis, the mechanical characteristics of the examined material is expressed as a function of the average $\mathrm{RH}$ and UPV values and there are published models in the literature for the estimation of the compressive strength of conventional concrete [45-48].

\section{To date there are very limited studies on the use of NDT techniques for the evaluation of the} mechanical performance of UHPFRC [12-13] and there are not any published studies on combined NDT methods (i.e. SonReb). The main aim of this study is to evaluate the reliability of NDT methods for the assessment of the mechanical characteristics (compressive strength and modulus of Elasticity) of UHPC and UHPFRC. Various mixes have been examined, with and without steel fibres (UHPFRC 
and UHPC), and compressive and tensile tests have been conducted alongside with RH and UPV tests at different ages. The application of established relationships for the correlation of the dynamic modulus of Elasticity with the UPV for homogeneous isotropic elastic medium have been examined $[12,13]$ and the results have been compared to the experimental values. The experimental results have been used for the development of NDT models using UPV, RH and combination of UPV and RH (SonReb) values.

\section{Experimental procedure}

\subsection{Preparation of UHPFRC}

In the current study, three different mixes have been examined; one using $1 \%$ steel fibres (UHPFRC1), one using 3\% steel fibres (UHPFRC-3) and another one without steel fibres (UHPC). UHPFRC mix design is based on previous studies $[5,7]$ where $2 \%$ and $3 \%$ (by volume of the mix) steel fibres have been used. In the current study the selection of the three mix designs of Table $1(0 \%, 1 \%$ and $3 \%$ steel fibres) has been made in order to evaluate the effect of the steel fibres quantity by comparing the results of mixes without steel fibres (UHPC), with low (UHPFRC-1) and with relatively high percentage of steel fibres (UHPFRC-3). All three concrete mix designs are presented in Table 1.

Silica fume, silica sand, Ground Granulated Blast Furnace Slag (GGBS), and cement class 32.5 R type II have been used together with polycarboxylate superplasticizer. Steel fibres with $13 \mathrm{~mm}$ length, diameter $0.16 \mathrm{~mm}$, tensile strength $3000 \mathrm{MPa}$, and modulus of Elasticity equal to $200 \mathrm{GPa}$ have also been incorporated in the UHPFRC mixes.

Regarding the mixing method, high-shear pan mixer (Zyklos ZZ $75 \mathrm{HE}$ ) has been used for all the examined mixes. Dry ingredients have been mixed first, and then superplasticizer has been added in the mix followed by the gradual addition of steel fibres through sieving. All specimens have been placed in a water curing tank until the testing day.

Cubic specimens with dimension $100 \mathrm{~mm}$ have been tested under compression alongside with nondestructive tests (RH and UPV) at 1, 2 (for UHPFRC-1 only), 3, 714 (for UHPFRC-1 only), and 28 days after casting. For the evaluation of the modulus of Elasticity and the tensile strength, dog-bone specimens have been cast and tested at 1, 2 (for UHPFRC-1 only), 3, 7 and 28 days. These testing ages have been selected in order to obtain a wide range of experimental results which will be able to be used for the regression analyses and for the development of empirical models. Three specimens have been tested for each mix and for all the examined ages. Geometry of dog-bone specimens is illustrated in Fig. 1a and cube and dog-bone samples after casting are presented in Fig. 1 b.

\subsection{Mechanical and Non-Destructive Testing}

The compressive strength tests were carried out using an Avery Denison compressive testing machine and the tests were conducted in accordance with BS EN 12390-3:2009 [49] with a loading rate of $0.6 \mathrm{MPa} / \mathrm{sec}$ (Fig. 2a). For the tensile testing of the dog-bone shaped specimens, tests under displacement rate of $0.007 \mathrm{~mm} / \mathrm{s}$ were conducted using an Instron 
universal testing machine. Linear Variable Differential Transformer (LVDT) and a special setup designed in order to measure the average of the extensions of the two sides of the specimens (Fig. 2b) [8]. The accuracy of the strain measurements has been validated using Digital Image Correlation (DIC) system and these values together with the load recordings have been used for the evaluation of the modulus of Elasticity values.

For the rebound hammer testing, nine impacts were conducted at each specimen and the median value was calculated as proposed by IS 13311 [18] and BS EN 12504-2 [50]. SilverSchmidt concrete rebound hammer was used in the current study. Tests were conducted on the moulded surfaces and all readings were taken at a distance not nearer on edge than $20 \mathrm{~mm}$ and not less than $20 \mathrm{~mm}$ from each other $[17,18]$ and the square of the coefficient of restitution values (Q-values) have been recorded. For the UPV measurements, Pundit Lab was used and the two transducers were placed on opposite sides of the cubes using a thin layer of couplant at the interface between the transducers and the specimens as proposed by BS 1881-203 [19] for the determination of pulse velocity by direct transmission. Three specimens have been examined for each mix for all the examined ages and these results have been used to correlate NDT characteristics to the respective compressive strength values.

\subsubsection{Mechanical testing results}

The development of compressive and tensile strength with the age of the specimens for the mixes with and without steel fibres, are presented in Fig. 3 and 4.

Results of Fig.3 indicate that, as expected, addition of 3\% of steel fibres leads to an increment of compressive strength values which is clear after 7 days of curing and is almost $5 \%$.

From the results of Fig.4 it is obvious that modulus of Elasticity and tensile strength are increased with the age of the specimens in all three mixes while the modulus of Elasticity values are not considerably affected by the addition of steel fibres. It is also evident that addition of $3 \%$ of steel fibres leads to a significant improvement of the post-cracking performance of the material. The slope of the initial linear part of the stress strain distributions (Fig. 4) have been used for the calculation of the modulus of Elasticity of the various mixes at all the examined ages. From the experimental results for the mix design without steel fibres (UHPC), seven modulus of Elasticity values have been calculated since in some of the examined specimens the failure occurred near the grips of the testing machine and these results have been eliminated from the calculations. For UHPFRC-1, thirteen modulus of Elasticity values have been calculated since two tests has been eliminated for the same reason. In case of UHPFRC-3 where high percentage of fibres has been used, all 12 tests have been successfully tested and all the results have been used for the calculation of modulus of Elasticity values.

\subsubsection{Application of theoretical model for homogeneous elastic medium}

The application of elastodynamic theory has been investigated and the reliability of the examined models has been validated using the experimental data. Based on previous studies $[12,13]$ the 


$$
\begin{aligned}
& \mathrm{V}_{\mathrm{p}, \mathrm{s}}=\sqrt{\frac{\mathrm{E}_{\mathrm{d}, \mathrm{u}}}{\rho} \frac{\left(1-\mathrm{v}_{\mathrm{u}}\right)}{\left(1+\mathrm{v}_{\mathrm{u}}\right)\left(1-2 \mathrm{v}_{\mathrm{u}}\right)}} \\
& \mathrm{E}_{\mathrm{cm}}=1.25 \mathrm{E}_{\mathrm{d}, \mathrm{u}}-19 \\
& \mathrm{E}_{\mathrm{cm}}=9500 *\left(\mathrm{f}_{\mathrm{c}}\right)^{1 / 3}
\end{aligned}
$$

152 (Fig. 7).

application of the established relationships for the correlation of the dynamic modulus of Elasticity $\left(E_{d, u}\right)$ and the UPV for homogeneous isotropic elastic medium are appropriate for the estimation of the elastic properties of UHPFRC since the material exhibits isotropic elastic behaviour [12, 13]. The theory of an ultrasonic pulse travelling in a homogenous isotropic elastic medium has been adopted in this study in order to correlate compressive wave velocity $\left(\mathrm{V}_{\mathrm{p}, \mathrm{s}}\right)$ with dynamic modulus of Elasticity $\left(\mathrm{E}_{\mathrm{d}, \mathrm{u}}\right)$, density $(\rho)$, and Poisson's ratio $\left(\mathrm{v}_{\mathrm{u}}\right)(\mathrm{Eq} .1)$ [51]. The model proposed by BSI CP110: [52] has been used to calculate the static modulus of Elasticity $\left(\mathrm{E}_{\mathrm{cm}}\right)$ from the dynamic modulus of Elasticity $\left(\mathrm{E}_{\mathrm{d}, \mathrm{u}}\right)$ (Eq. 2). The compressive strength $\left(\mathrm{f}_{\mathrm{c}}\right)$ has also been calculated from the values of the static modulus of Elasticity $\left(\mathrm{E}_{\mathrm{cm}}\right)$ using the model proposed by Eurocode 2 (Eq. 3) [53].

Eq. 1-3 have been used to calculate static modulus of Elasticity $\left(\mathrm{E}_{\mathrm{cm}}\right)$ and compressive strength using the ultrasonic wave velocity values $\left(\mathrm{V}_{\mathrm{p}, \mathrm{s}}\right)$. In the current study average Poisson's ratio value of $\mathrm{v}_{\mathrm{u}}=0.2$ has been used [13] while the density values which are based on experimental measurements have been taken equal to $2187 \mathrm{~kg} / \mathrm{m}^{3}$ for UHPC, $2244 \mathrm{~kg} / \mathrm{m}^{3}$ for UHPFRC-1, and $2357 \mathrm{~kg} / \mathrm{m}^{3}$ for UHPFRC-3. Using these parameters, the static modulus of Elasticity has been calculated using Eq. 1-2and the results are compared with the respective experimental values (Fig. 5).

The results of Fig. 5 have been used for the calculation of the error (\%) in the modulus of Elasticity values $\left(\mathrm{E}_{\mathrm{cm}}\right)$ calculated for UHPC, UHPFRC-1 and UHPFRC-3. The distribution of the error (\%) with the different values of the modulus of Elasticity is presented in Fig. 6.

The results of Fig. 6 indicate that overall the error is reduced as the modulus of Elasticity values are increased. This can be attributed to the fact that Eq. 2 have been derived for conventional concrete and calibration of this model is required for the calculation of the early-age characteristics.

The theoretical results for the modulus of Elasticity and Eq.3 have been used for the calculation of the compressive strength values and these results are compared with the respective experimental results

The results presented in Fig. 7 indicate that application of elastodynamic theory together with Eq. 3 cannot accurately predict the actual compressive strength values of UHPFRC. This is attributed to limitation of Eq. 2 and Eq. 3 which have been empirically calculated based on data for conventional concrete. Also based on the results of Fig. 7, it is evident that the theoretical equations lead the considerably lower values compared to the experimental results. The theoretical values are lower than half of the respective experimental values and the deviation between the experimental and the 
theoretical values is not considerably affected by the percentage of the steel fibres since it is almost the same for UHPC, UHPFRC-1 and UHPFRC-3. In the following sections, the development of appropriate empirical relationships for the calculation of the compressive strength and the modulus of Elasticity based on the NDT results will be presented.

\subsubsection{Non-destructive results versus mechanical testing: Empirical models}

In this section, empirical relationships between the results of mechanical and non-destructive tests, have been developed. Empirical models for conventional concrete have been proposed in previous studies for the calculation of the compressive strength using RH and UPV measurements where experimental results have been used and best fit lines have been calculated [26, 38]. Also, the strong correlation between the modulus of Elasticity $\left(\mathrm{E}_{\mathrm{cm}}\right)$ and the UPV has been highlighted in a previous study where the use of empirical models for the calculation of $E_{c m}$ of conventional and sub-standard concretes has been proposed as a simplified method [39]. Linear and exponential regression models have been examined in the current study for the evaluation of the compressive strength and modulus of Elasticity.

\subsubsection{RH and UPV versus compressive strength results-Linear and Exponential models}

The relationship between $\mathrm{Q}$ and compressive strength values together with the linear and exponential regression models and the coefficient of determination $\left(\mathrm{R}^{2}\right)$ for UHPC (without steel fibres), UHPFRC-1 and UHPFRC-3 are presented in Fig. 8a, Fig. 8b and Fig. 8c respectively.

According to the results of Fig. 8, high coefficient of determination values for both linear and exponential regression models have been found for all the examined cases. In both linear and exponential regression models, the coefficient of determination has been found to be in the range 0.940.98. In case of the mix without steel fibres (UHPC), similar values of the coefficient of determination for linear and exponential models have been obtained (0.94 and 0.95). For specimens with $1 \%$ steel fibres (UHPFRC-1), higher coefficient of determination have been observed for linear regression model while for specimens with $3 \%$ steel fibres (UHPFRC-3), higher value of the coefficient has been observed for exponential regression.

The compressive strength results versus UPV are presented in Fig. 9 together with respective linear and exponential regression models for UHPC, UHPFRC-1 and UHPFRC-3. The results indicate that in all the examined cases, exponential regression models have been found to have very high values almost equal to unity (0.99-1.00). In case of linear regression models, the coefficients of determination were found to be in the range 0.90-0.97 (Fig. 9).

In the following section, NDT results are compared to the modulus of Elasticity values and respective empirical linear and exponential models have been calculated. 
209

210

211

212

213

214

215

216

217

218

219

220

221

222

223

224

225

226

227

228

229

$F=a V^{b} S^{c}$

where:

$F \quad$ is the mechanical characteristic (compressive strength or Modulus of Elasticity,

$V \quad$ is the ultra-sonic pulse velocity,

$S \quad$ is the Q-value from the rebound hammer tests, and

$a, b, c$ are constants depended on the material.

231 The natural logarithms of the data of Fig. 8 and Fig. 9 have been initially calculated, and then

232 'LINEST' function in Microsoft Excel has been used to calculate straight lines to best fit the data 233 using the 'least squares' following the procedure proposed by Proceq [54]. Based on these analyses, 234 appropriate values for coefficients $\mathrm{a}, \mathrm{b}, \mathrm{c}$ for the compressive strength of UHPC and UHPFRC 1 and 235 UHPFRC 3 have been determined (Table 2). Also, the respective values proposed by RILEM 43-CND $236[45]$ for standard concretes are presented in the same table. 
The same analyses have been conducted for the modulus of Elasticity using the results presented in

238

239

240

241

242

243

244

245

246

247

248

249

250

251

252

253

254

255

256

257

258

259

260

261

262

263

264

265

266

267

268

269 Fig. 9 and Fig. 10 and SonReb coefficients for the modulus of Elasticity are presented in Table 3. The SonReb models with the coefficients calculated in this study (Tables 2 and 3) have been applied for various UPV and Q values and the results of the compressive strength and modulus of Elasticity are presented in Fig. 12a and Fig. 12b respectively.

From the results of Fig. 12 it is obvious that as expected, compressive strength and modulus of Elasticity are both increased as UPV and Q values are increased. The results show that the trend of the models is almost the same for all the examined mixes but the exact values are highly affected by the various mix designs that have been examined (UHPC, UHPFRC-1, and UHPFRC-2), so appropriate calibration is required for different mixes. Only in case of compressive strength values (Fig. 12a) the results have been found to be quite similar for UHPFRC-1 and UHPFRC-3 mixes.

In order to evaluate the empirical models of Table 2, isoresistance curves for all the examined mixes (UHPC, UHPFRC-1 and UHPFRC-3) have been generated and these results have been compared with the respective curves for conventional concrete using the model proposed by RILEM 43-CND [45] (table 2). The isoresistance curves for all the examined models are presented in Fig. 13.

From the results of Fig. 13 it is evident that the mix design is considerably affecting the shape of the isothermal curves. For high performance mix design without steel fibres (UHPC) an abrupt drop of the Q-Value is observed as UPV is increased, while as the fibre percentage is increased (UHPFRC-1 and UHPFRC-3), this drop becomes more gradual. The shapes of the models for UHPFRC-1 and UHPFRC-3 are similar to the shape of RILEM 43-CND [45] model for the examined range of values (Fig. 13). However it is clear that RILEM 43-CND [45] model for conventional concrete leads to considerably lower compressive strength values. This is attributed to the special mixture composition in case of UHPC and UHPFRC and mainly in the high cement content, in the aggregates type (silica sand) and in the addition of admixtures (slag and silica fume used in UHPFRC). These factors are crucial for the parameters of the empirical SonReb models [45]. According to RILEM 43-CND [45] correction factors (coefficients of influence) should be applied in order to calibrate the model for different mix designs. In the examined mixes with UHPFRC-1 and UHPFRC-3 coefficients of influence in the range of 1.5-2 should be used in order to take into account the effect of the special composition of the examined mixes.

In the following section (Section 2.3), the accuracy of the calculated SonReb empirical models is compared with the accuracy of the linear and exponential models presented in 2.2.3.1 and 2.2.3.2.

2.3 Evaluation of the accuracy of linear, exponential and combined SonReb models for the calculation of compressive strength and modulus of Elasticity values

In order to evaluate the accuracy of all the examined models, the results of the linear, the exponential and the combined SonReb empirical models have been compared with the actual mechanical tests results. The mean values of the calculated results for the various mixes (UHPC, UHPFRC 1, UHPFRC 3 ) at all the examined ages are compared with the respective compressive strength and modulus of 
Elasticity results obtained from the mechanical testing, and the comparisons are presented in Fig. 14 and Fig. 15 together with the diagonal line (line of equality) which represents absolute equality.

276

277

278

279

280

281

282

283

284

285

286

287

288

289

290

291

292

293

294

295

296

297

298

299

300

301

302

303

304

305

306

307

308

309

The results of Fig. 14 and Fig. 15 indicate that in all the examined cases the values calculated with the combined SonReb method are very close to the line of equality for the whole range of the examined values and for both compressive strength and modulus of Elasticity values. In order to quantify the level of accuracy of each method, the \% difference (error) between the predicted value using each of the methods and the respective actual value obtained from mechanical tests, has been calculated. In Fig. 16 and Fig. 17, errors (\%) versus actual mechanical characteristics obtained from the mechanical tests are presented for each mix design.

From the compressive strength results presented in Fig. 16, it is obvious that the lowest error values for UHPC and UHPFRC-1 have been achieved for the combined SonReb. For UHPFRC-3 the lowest error values for compressive strength values up to $75 \mathrm{MPa}$ have been calculated using Exponential-Q while for higher strength values SonReb and Exponential-V models have been found to be the most accurate ones. For all the examined cases the error (\%) values of SonReb method have been found to be below 10\%. Overall the highest error values have been observed for the Exponential-Q and Linear$\mathrm{V}$ models for UHPC and UHPFRC-1, while for UHPFRC-3, Linear-V and Linear-Q have been found to give the highest error values. Exponential- $\mathrm{V}$ model has been found to give very low error values and below $10 \%$ in case of UHPFRC-1 and UHPFRC-3. However, the error value was quite high and exceeded $10 \%$ in case of mix design without steel fibres (UHPC).

Regarding the modulus of Elasticity results (Fig. 17), overall the most accurate values have been calculated with the combined SonReb method. All the error values of SonReb method have been found to be below $1.2 \%$ for UHPC, below $5.7 \%$ for UHPFRC-1, and below $8.6 \%$ for UHPFRC-3 for modulus of Elasticity values lower than $30 \mathrm{MPa}$ while for higher values the error has not exceeded $1 \%$. Exponential-Q models have been found to lead to very low error values and below $5.7 \%$ for UHPC and UHPFRC-3 mixes, but high error values in the range of 5.9-19.0\% have been observed for UHPFRC-1. The highest error values overall have been observed for Linear-Q models.

From the observations described above it is evident that the only model which provides accurate results with low error values (below 10\%) in all the examined cases is SonReb. For all the other models, there are variations in the degree of accuracy depending on the mix design and the special characteristics of each specimen. This is attributed to the random distribution of the various components of the mix and especially to the dispersion of the steel fibres which leads to a variation in the properties of the materials and especially at the surface of the material. Parameters such as the mixing procedure and the vibration of the material can considerably affect the characteristics of the outer surface and subsequently its hardness. In this case, Rebound Hammer (RH) measurements which are mainly affected by the hardness of the surface are not sufficient for the accurate evaluation of the mechanical characteristics of the material, and combination with UPV measurements (i.e. SonReb) is 
Elasticity of UHPFRC mixes. This is evidenced by all the results presented in the current study.

312

313

314

315

316

317

318

319

320

321

\section{Conclusions}

In the current study, the reliability of NDT methods for the evaluation of the compressive strength and the modulus of Elasticity of UHPC and UHPFRC has been investigated. Ultrasonic Pulse Velocity (UPV), Rebound Hammer (RH) and combined SonReb techniques have been examined and various models have been developed and evaluated using actual mechanical characteristics obtained from the mechanical tests. Also, the reliability of theoretical models for homogenous elastic medium for the calculation of the modulus of Elasticity of UHPC and UHPFRC using UPV measurements has been evaluated.

Based on the findings of this investigation, the following conclusions have been drawn.

Effect of steel fibres on mechanical characteristics of UHPFRC:

- Based on the mechanical testing, the compressive strength of UHPFRC with 3\% steel fibres is slightly higher (almost 5\%) compared to the respective values obtained for UHPC (without steel fibres).

- Modulus of Elasticity is not considerably affected by the addition of steel fibres, while the post-cracking response of the material is significantly enhanced as the steel fibres percentage is increased.

Evaluation of the examined NDT techniques:

- From the application of the theoretical models for homogenous elastic medium it has been observed that the static modulus of Elasticity cannot be accurately predicted especially for relatively low values, due to the fact that Eq. 2 has been empirically calculated based on data for conventional concrete. Also, the application of the theoretical model (Eq. 1) together with the empirical relationships (Eq. 2 and Eq. 3) lead to considerably lower compressive strength values compared to the respective experimental results.

- From the correlation of compressive strength with NDT values, high coefficient of determination values (0.94-0.98) for both linear and exponential regression models have been found for Q-values versus compressive strength. From the distribution of UPV with compressive strength results, coefficient of determination values in the range of 0.90-0.94 have been found for linear regression models, while significantly higher are the respective values (0.98-0.99) for exponential regression models.

- Regarding the $\mathrm{Q}$ versus modulus of Elasticity values, lower regression values compared to the respective results for the compressive strength have been calculated for both linear and exponential models (0.81-0.88 for linear regression models and 0.78-0.94 for exponential models). From the correlation of UPV with the modulus of Elasticity, coefficients of 
determination in the range of 0.71-0.97 have been calculated for linear regression values, while overall higher values (0.82-0.95) have been achieved for exponential models.

- Isoresistance curves for the proposed models for UHPC, UHPFRC-1, UHPFRC-3 have been compared with the proposed model by RILEM 43-CND [45] for conventional concrete. From

\section{Acknowledgments}

The authors would like to greatly acknowledge Sika Limited and Hanson Heidelberg Cement Group for providing raw materials for the experimental part of this study. Also, the authors would like to express their gratitude to the anonymous reviewers for their comments who have led to significant improvement of the quality of the paper. 


\section{References}

[1] B.A. Graybeal, Material property characterization of ultra-high performance concrete, Federal Highway Administration (2006).

[2] S.T. Kang, Y. Lee, Y.D. Park, J.K. Kim, Tensile fracture properties of an Ultra High Performance Fiber Reinforced Concrete (UHPFRC) with steel fiber, Composite Structures, 92(1) (2010) 6171.

[3] Y. Yoo, H.O. Shin, J.M. Yang, Y.S. Yoon, Material and bond properties of ultra high performance fiber reinforced concrete with micro steel fibers, Composites Part B: Engineering, 58 (2013) $22-133$.

[4] S.T. Kang, J.K. Kim, The relation between fiber orientation and tensile behavior in an Ultra High Performance Fiber Reinforced Cementitious Composites (UHPFRCC), Cement and Concrete Research, 41(10) (2011) 1001-1014.

[5] A. Hassan, S. Jones, G. Mahmud, Experimental test methods to determine the uniaxial tensile and compressive behaviour of ultra-high performance fibre reinforced concrete (UHPFRC), Construction and Building Materials, 37 (2012) 874-882.

[6] R. Toledo Filho, E. Koenders, S. Formagini, E. Fairbairn, Performance assessment of ultra-high performance fibre reinforced cementitious composites in view of sustainability, Materials \& Design, 36 (2012) 880-888.

[7] A.P. Lampropoulos, S.A. Paschalis, O.T. Tsioulou, S.E. Dritsos, Strengthening of reinforced concrete beams using ultra high performance fibre reinforced concrete (UHPFRC), Engineering Structures, 106 (2016) 370-384.

[8] A.P. Lampropoulos, S.A. Paschalis, Ultra High Performance Fiber Reinforced Concrete Under Cyclic Loading, ACI Material Journal, 113 (2016) 419-427.

[9] D. Nicolaides, A. Kanellopoulos, P. Savva, and M. Petrou, Experimental field investigation of impact and blast load resistance of Ultra High Performance Fibre Reinforced Cementitious Composites (UHPFRCCs), Construction and Building Materials, 95 (2015) 566-574.

[10] BFUP AFGC, Ultra high performance fibre-reinforced concretes, Interim recommendations, AFGC/SETRA Working Group, France (2002).

[11] A.A. Shah, Y. Ribakov, Recent trends in steel fibered high-strength concrete, Materials \& Design, 32(8-9) (2011) 4122-4151.

[12] G. Washer, P. Fuchs, B.A. Graybeal, J.L. Hartmann, Ultrasonic testing of reactive powder concrete, IEEE Trans Ultrason Ferroelectr Frequency Control, 51(2) (2004) 193-201. 
[13] A. Hassan, S. Jones, Non-destructive testing of ultra high performance fibre reinforced concrete (UHPFRC): A feasibility study for using ultrasonic and resonant frequency testing techniques, Construction and Building Materials, 35 (2012) 361-367.

[14] V. M. Malhotra, Testing Hardened Concrete: Non-destructive Methods, ACI, monograph No. 9, Detroit (1976).

[15] A. Leshchinsky, Non-destructive methods instead of specimens and cores, quality control of concrete structures, International Symposium Held by RILEM. Belgium (1991).

[16] K. Themistocleous, K. Neocleous, K. Pilakoutas, D. G. Hadjimitsis, Damage assessment using advanced non-intrusive inspection methods: integration of space, UAV, GPR, and field spectroscopy, in: Second International Conference on Remote Sensing and Geoinformation of the Environment, Pafos, 2014, 7-10 April, 2014.

[17] BS1881-202, Recommendations for surface hardness testing by rebound hammer, BSI, UK (1986).

[18] IS 13311 (Part I): 1992 Non-Destructive Testing of Concrete Methods of test(Ultrasonic Pulse Velocity), BIS, New Delhi (1992).

[19] BS 1881-203, Recommendations for measurement of velocity of ultrasonic pulses in concrete, BSI, UK (1986).

[20] I.N. Prassianakis, P. Giokas, Mechanical properties of old concrete using destructive and ultrasonic non-destructive testing methods, Magazine of Concrete Research, 55 (2003) 171-176.

[21] J.H. Bungey, The validity of ultrasonic pulse velocity testing of in-place concrete for strength, N.D.T. International 13(6) (1980) 296-300.

[22] I. Facaoaru, Contribution à i'étude de la relation entre la résistance du béton à la compression et de la vitesse de propagation longitudinale des ultrasons, RILEM 22 (1961) 125-154. (in French)

[23] K.G. Trezos, K. Georgiou, C. Marebelias, Determination of the in situ strength of concrete using the indirect methods of impact and the ultrasounds, Technika Chronika-Scientific Edition TCG 13 (1993) 27-41.

[24] D. Mikulic, Z. Pause, V. Ukrainc, Determination of concrete quality in a structure by combination of destructive and non-destructive methods, Materials and Structures 25 (1992) 65-69.

[25] L. Logothetis, Combination of three non-destructive methods for the determination of the strength of concrete, PhD Thesis, National Technical University of Athens (1979).

[26] H.Y. Qasrawi, Concrete strength by combined non-destructive methods simply and reliably predicted, Cement and Concrete Research, 30 (2000) 739-746. 
[27] R.S. Ravindrarajah, Y.H. Loo and C.T. Tam, Recycled concrete as fine and coarse aggregates in concrete, Magazine of Concrete Research, 39 (1987) 214-220.

[28] A. Goncalves, In situ concrete strength estimation. Simultaneous use of cores, rebound hammer and pulse velocity, International Symposium NDT in Civil Engineering, Germany (1995) $977-$ 984.

[29] T. Soshiroda, K. Voraputhaporn, Recommended method for earlier inspection of concrete quality by non-destructive testing, Concrete Durability and Repair Technology (1999) 27-36.

[30] K.K. Phoon, T.H. Wee, C.S. Loi, Development of statistical quality assurance criterion for concrete using ultrasonic pulse velocity method, ACI Material Journal, 96 (5) (1999) 568-573.

[31] R.H. Elvery, LAM. Ibrahim, Ultrasonic assessment of concrete strength at early ages, Magazine of Concrete Research, (1976) 181-190.

[32] G.V.Teodoru, The use of simultaneous nondestructive tests to predict the compressive strength of concrete, ACI SP-112, (1998) 137-152.

[33] Y. Tanigawa, K. Baba, H. Mori, Estimation of concrete strength by combined nondestructive testing method, ACI SP 82, (1984) 57-76.

[34] W.E. Parker, Pulse velocity testing of concrete, Proceedings - American Society for Testing Materials, 53 (1953) 1033-1042.

[35] C.H. Yun, K.R. Choi, S.Y. Kim, Y.C. Song, Comparative evaluation of nondestructive test Methods for in-place strength determination, ACI SP 112 (1988) 111-136.

[36] L.M. Rio, A. Jimenez, F. Lopez, F.J. Rosa, M.M. Rufo, J.M. Paniagua, Characterization and hardening of concrete with ultrasonic testing, Ultrasonics, 42 (2004) 527-530.

[37] E. Arioglu, N. Arioglu, Testing of concrete core samples and evaluations, Evrim Publisher, Istanbul (1998).

[38]G.F. Kheder, A two stage procedure for assessment of in-situ concrete strength using combined non-destructive testing. Materials and Structures, 32 (1998) 410-417.

[39]H. Y1ldırım, O. Sengul, Modulus of elasticity of substandard and normal concretes. Construction and Building Materials, 25 (2011) 1645-1652.

[40] M. K. Lim, H. Cao, Combining multiple NDT methods to improve testing effectiveness, Construction and Building Materials, 38 (2013) 1310-1315.

[41] G. Concu, B. De Nicolo, L. Pani, Non-Destructive Testing as a tool in reinforced concrete buildings refurbishments, Structural Survey, 29 (2) (2011) 147-161. 
482

483

[42] D. Breysse, Nondestructive evaluation of concrete strength: an historical review and a new perspective by combining NDT methods, Construction and Building Materials, 33 (2012) 139163.

[43] I.R. De Almeida, Non-destructive Testing of High Strength Concretes: Rebound (Schmidt Hammer and Ultrasonic Pulse Velocity), International Symposium held by RILEM, Belgium (1991) 387- 397.

[44] G. Kheder, Assessment of in Situ Concrete Strength Using Combined Non-destructive Testing, First International Arab Conference on Maintenance and Rehabilitation of Concrete Structures, Cairo (1998) 59-75.

[45] RILEM 43-CND, Combined non-destructive testing of concrete. Draft recommendation for in situ concrete strength determination by combined non-destructive methods, Materials and Structures, 26 (1993) 43-49.

[46] R. Giochetti, L. Lacquaniti, Controlli non distruttivi su impalcati da ponte in calcestruzzo armato, Nota Tecnica 04, Università degli Studi di Ancona, Facoltà di Ingegneria, Istituto di Scienza e Tecnica delle Costruzioni (1980). (in Italian)

[47] J. Gasparik, Prove non distruttive in edilizia, Quaderno didattico A.I.P.n.D., Brescia (1992). (in Italian)

[48] A. Di Leo, G. Pascale, Prove non distruttive sulle costruzioni in cemento armato, Convegno Sistema Qualità e Prove non Distruttive per l'affidabilità e la sicurezza delle strutture civili, Bologna, SAIE'94 (1994). (in Italian)

[49] BS EN 12390-3, Compressive Strength of Test Specimens, Testing Hardened Concrete, 1-22, BSI, UK (2009).

[50] BS EN 12504-2, Non-destructive Test. Determination of Rebound Number, BSI, UK (2001).

[51] R. Jones, Non-destructive testing of concrete, London: Cambridge University Press (1962).

[52] BSI CP110: Part 1, Code of Practice for the Structural Use of Concrete, BSI, UK (1972).

[53] European pre-standard: ENV 1992-1-1: Eurocode 2: Design of concrete structures - Part 1: General rules and rules for buildings.

[54] Proceq, Using EXCEL to determine SONREB curve coefficients, Switzerland. 


\section{List of tables}

Table 1. Mix design of UHPFRC.

\begin{tabular}{llll}
\hline & \multicolumn{3}{l}{ Mix proportions $\left(\mathrm{kg} / \mathrm{m}^{3}\right)$} \\
Material & UHPC & UHPFRC-1 & UHPFRC-3 \\
\hline Cement & 657 & 657 & 657 \\
GGBS & 418 & 418 & 418 \\
Silica fume & 119 & 119 & 119 \\
Silica Sand & 1051 & 1051 & 1051 \\
Superplasticizers & 59 & 59 & 59 \\
Water & 185 & 185 & 185 \\
Steel fibres & 0 & 75.2 & 235.5 \\
\hline
\end{tabular}

Table 2. SonReb coefficients for compressive strength models.

\begin{tabular}{ccccc}
\hline Coefficient & $\begin{array}{c}\text { Values calculated } \\
\text { for UHPC }\end{array}$ & $\begin{array}{c}\text { Values calculated for } \\
\text { UHPFRC-1 }\end{array}$ & $\begin{array}{c}\text { Values calculated } \\
\text { for UHPFRC-3 }\end{array}$ & $\begin{array}{c}\text { Values proposed by } \\
\text { RILEM 43-CND [45] }\end{array}$ \\
\hline $\mathrm{a}$ & $2.36 \mathrm{E}-20$ & $2.78 \mathrm{E}-11$ & $1.61 \mathrm{E}-08$ & $2.756 \mathrm{E}-10$ \\
$\mathrm{~b}$ & 5.80 & 3.00 & 2.28 & 2.487 \\
$\mathrm{c}$ & 0.23 & 0.92 & 0.87 & 1.311 \\
\hline
\end{tabular}

Table 3. SonReb coefficients for modulus of Elasticity models.

\begin{tabular}{|c|c|c|c|}
\hline \multirow{2}{*}{ Coefficient } & \multirow{2}{*}{$\begin{array}{l}\text { Values calculated } \\
\text { for UHPC }\end{array}$} & \multicolumn{2}{|c|}{ Values calculated for Values calculated for } \\
\hline & & UHPFRC 1 & UHPFRC 3 \\
\hline a & $1.12 \mathrm{E} 32$ & $4.05 \mathrm{E}-11$ & $1.11 \mathrm{E} 16$ \\
\hline$b$ & -11.00 & 3.43 & -5.73 \\
\hline$c$ & 5.72 & -0.37 & 3.84 \\
\hline
\end{tabular}




\section{List of figures' captions}

Fig. 1. a) Geometry of dog-bone specimens and b) dog-bone and cube samples.

Fig. 2. Experimental setup for a) compressive and b) tensile testing of the examined specimens.

Fig. 3. Compressive strength for specimens with and without steel fibres at various ages.

Fig. 4. Tensile strength vs strain at different ages for a) UHPC, b) UHPFRC-1 and c) UHPFRC-3.

Fig. 5. Comparisons between experimental and theoretical values of $\mathrm{E}_{\mathrm{cm}}$

Fig. 6. Distribution of error values (\%) with the modulus of Elasticity values for UHPC, UHPFRC-1 and UHPFRC-3.

Fig. 7. Comparisons between the theoretical and the experimental results of the compressive strength.

Fig. 8. Correlation of compressive strength with Q-values and respective linear and exponential regression models for a) UHPC, b) UHPFRC-1 and c) UHPFRC-3.

Fig. 9. Correlation of compressive strength with UPV results and respective linear and exponential regression lines for a) UHPC, b) UHPFRC-1 and c) UHPFRC-3.

Fig. 10. Correlation of modulus of Elasticity with Q-values and respective linear and exponential regression lines for a) UHPC, b) UHPFRC-1 and c) UHPFRC-3.

Fig. 11. Correlation of modulus of Elasticity with UPV results and respective linear and exponential regression lines for a) UHPC, b) UHPFRC-1 and c) UHPFRC-3.

Fig. 12. SonReb models a) for compressive strength and b) for modulus of Elasticity for all the examined mixes.

Fig. 13. Isoresistance curves for the proposed models for UHPC, UHPFRC-1, UHPFRC-3 and comparison with RILEM 43-CND [45] model for conventional concrete.

Fig. 14. Correlation between compressive strength values calculated using different models and actual compressive strength values for a) UHPC (without steel fibres), b) UHPFRC-1 and c) UHPFRC-3.

Fig. 15. Correlation between modulus of Elasticity values calculated using different models and actual compressive strength values for a) UHPC (without steel fibres), b) UHPFRC-1 and c) UHPFRC-3.

Fig. 16. Error (\%) for all the examined compressive strength models for a) UHPC, b) UHPFRC-1 and c) UHPFRC-3.

Fig. 17. Error (\%) for all the examined modulus of Elasticity models for a) UHPC, b) UHPFRC-1 and c) UHPFRC-3. 

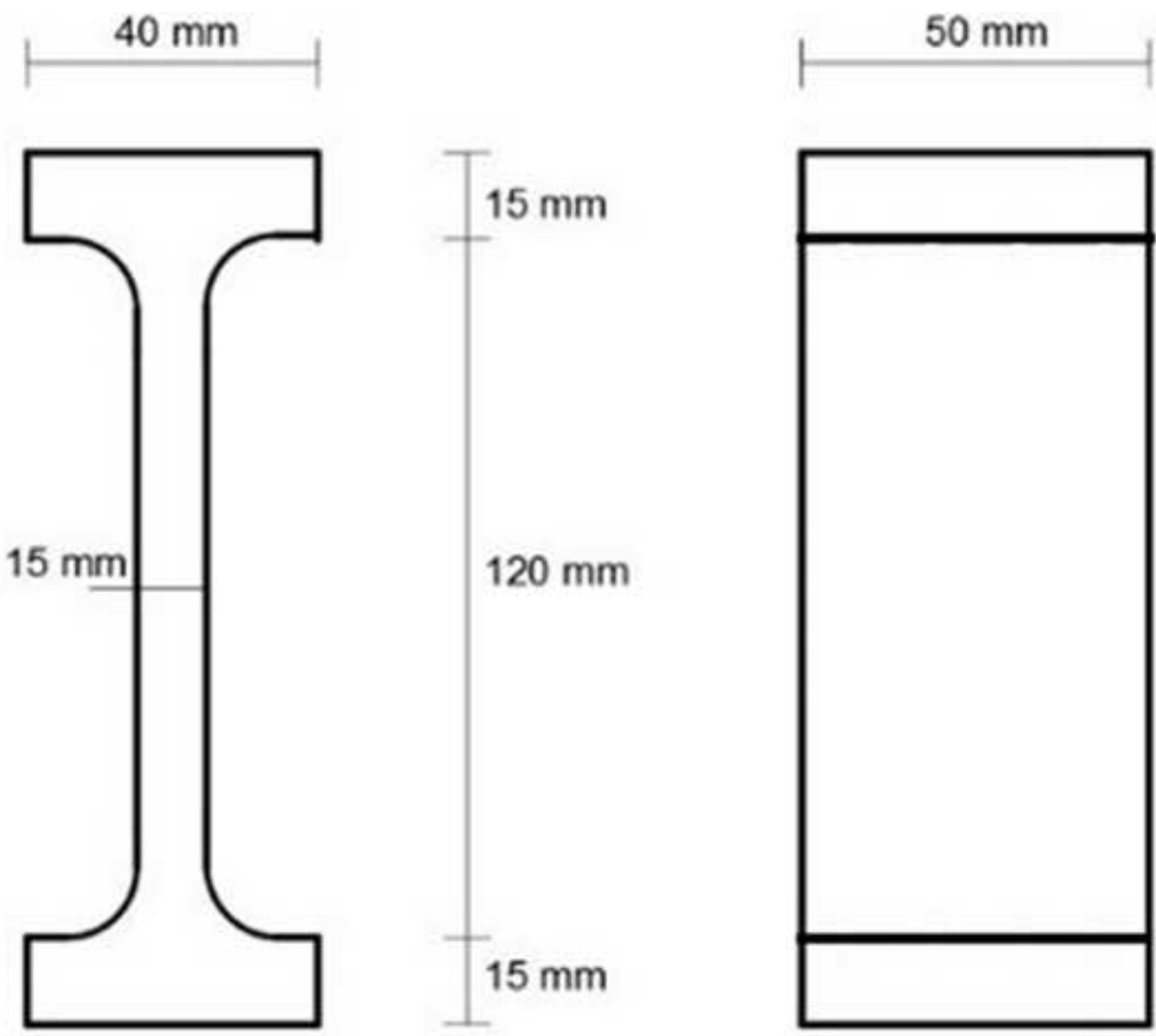

\section{Front view}

\section{Side view}

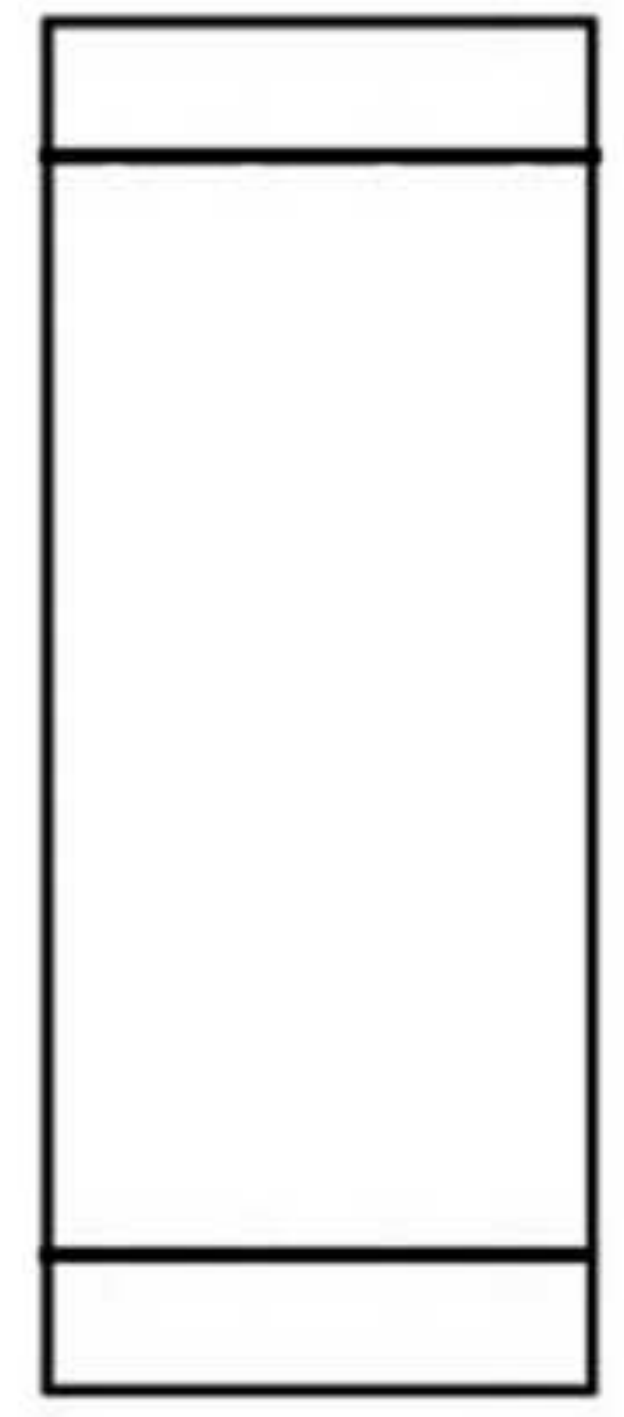


Click here to download high resolution image

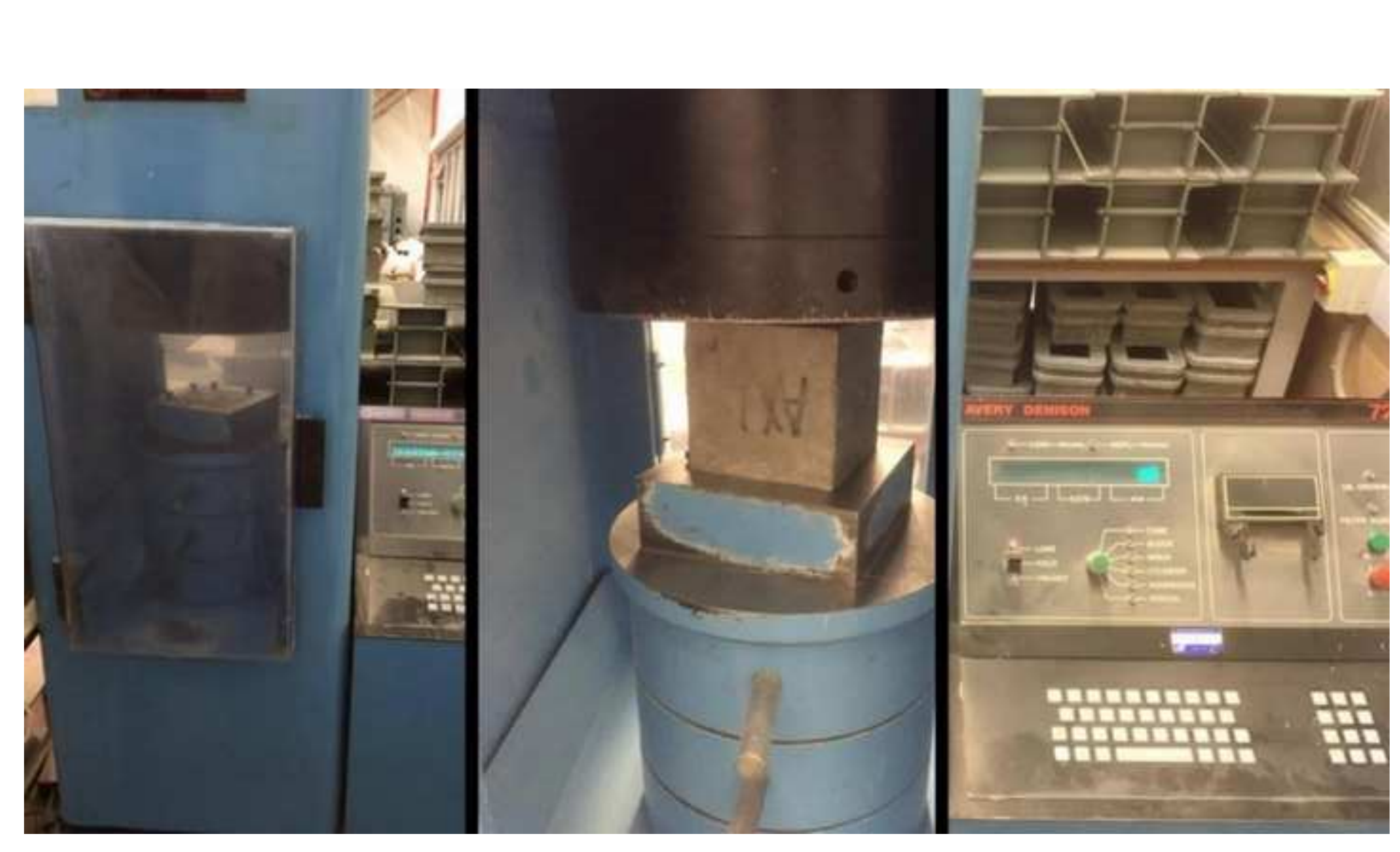

click here to download high resolution image 
Click here to download high resolution image

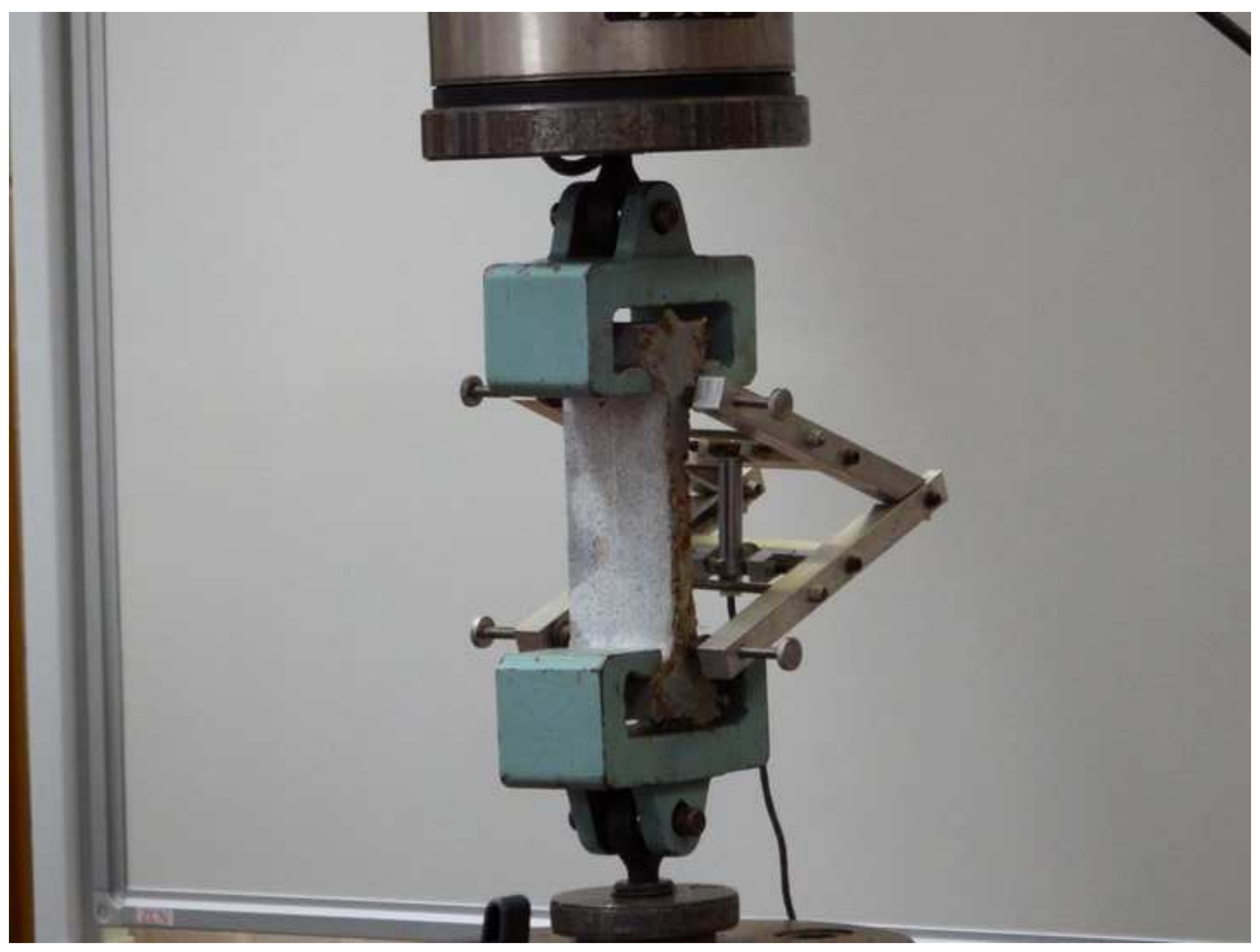




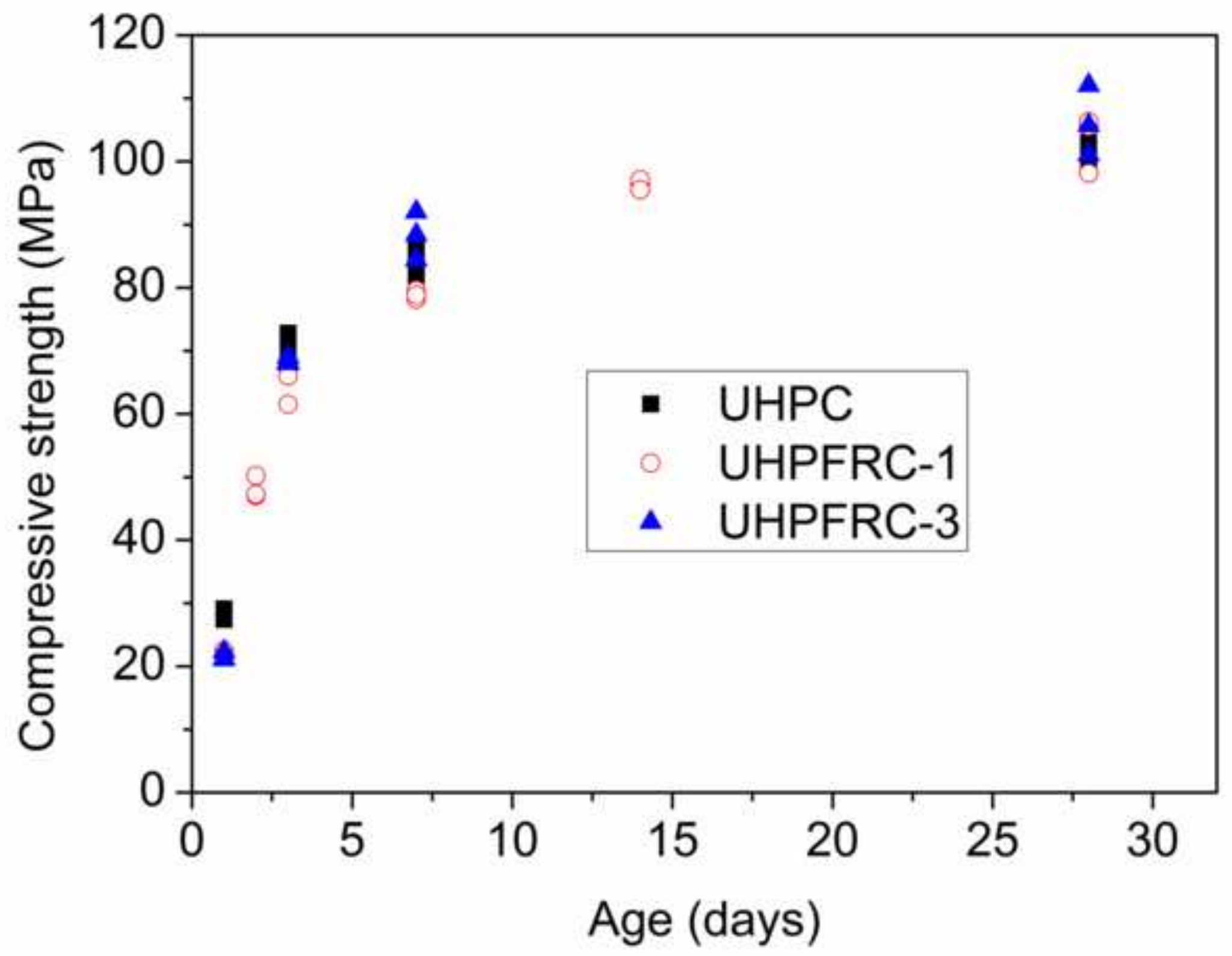




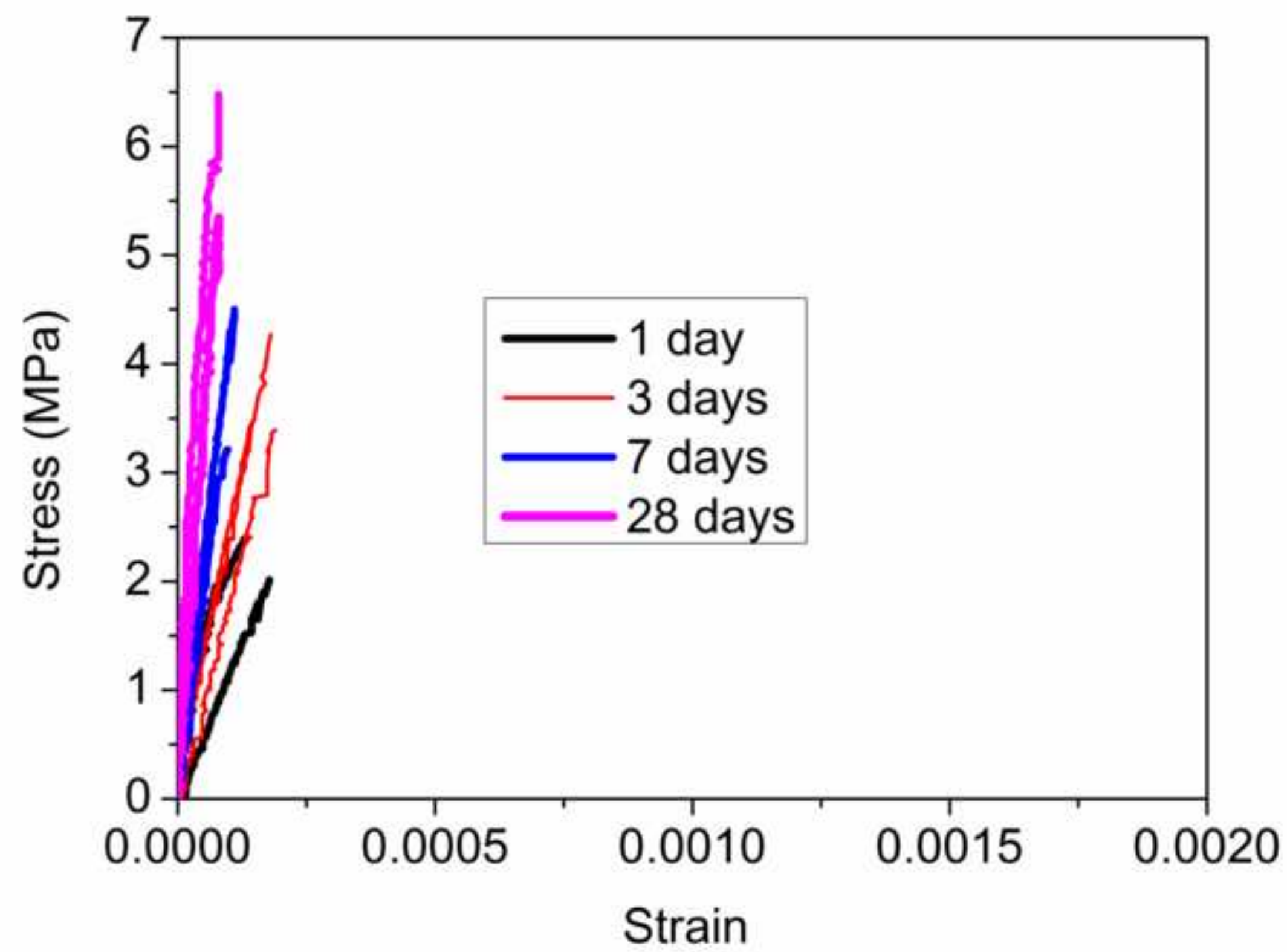




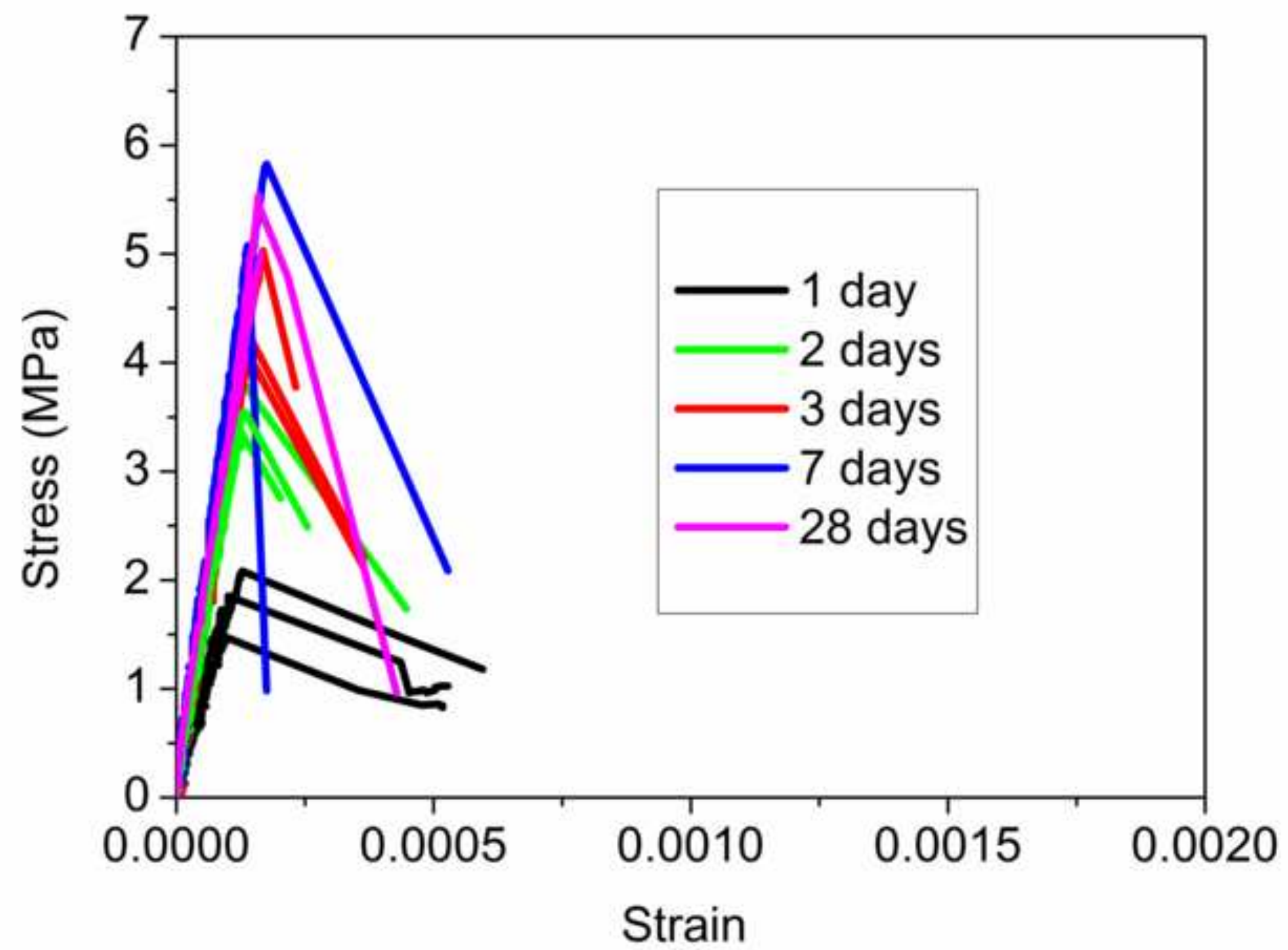




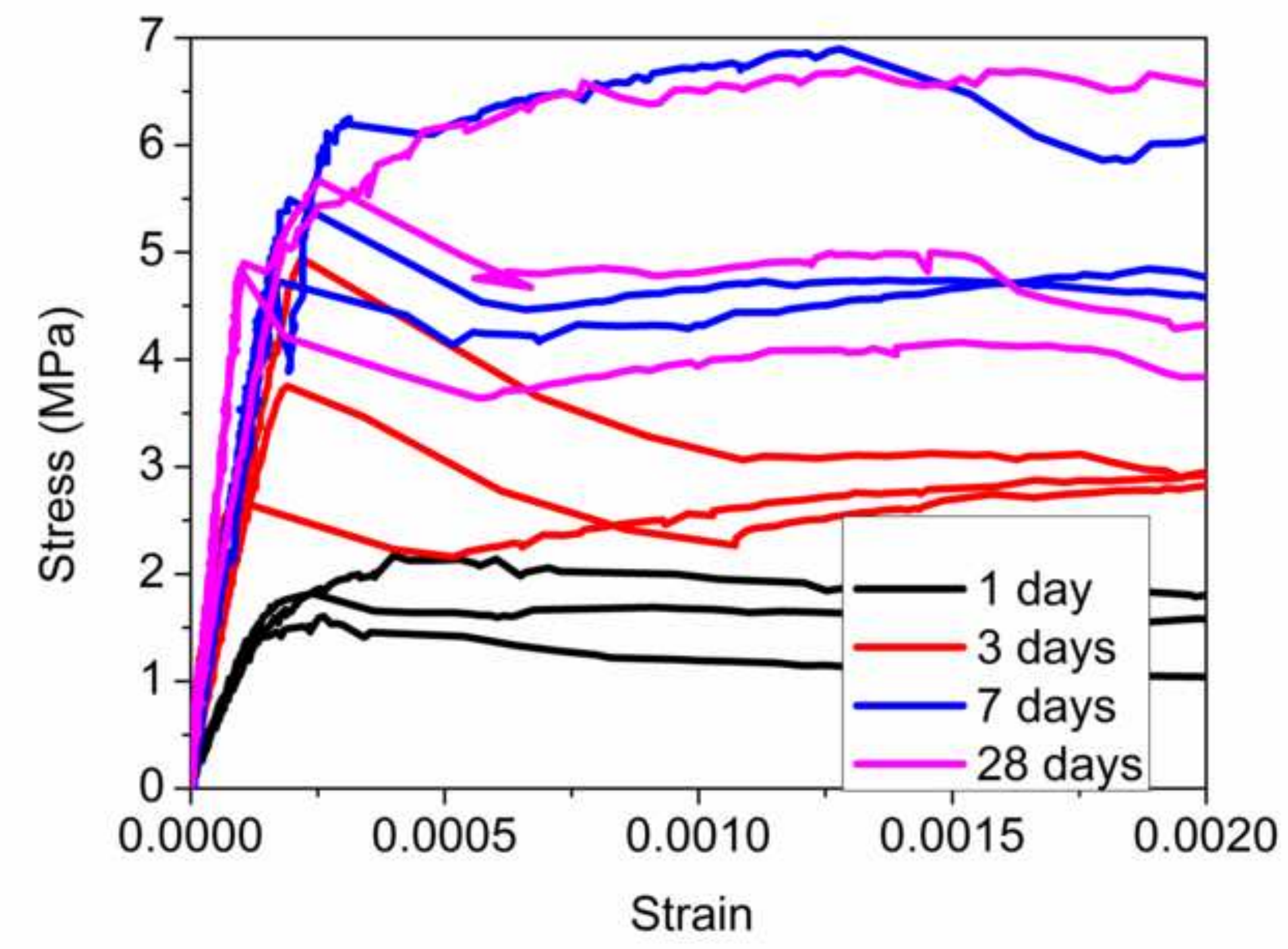

Click here to download high resolution image 


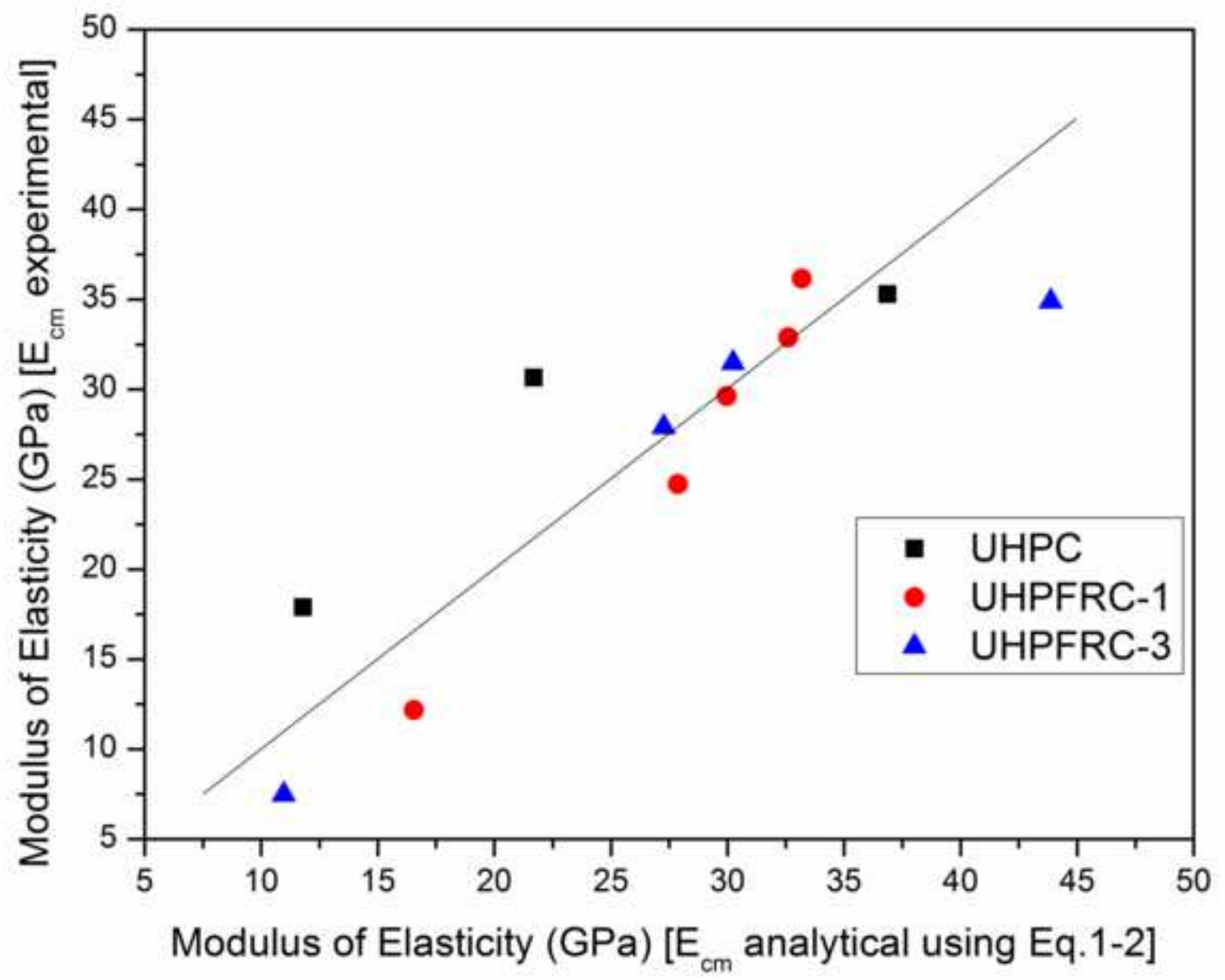




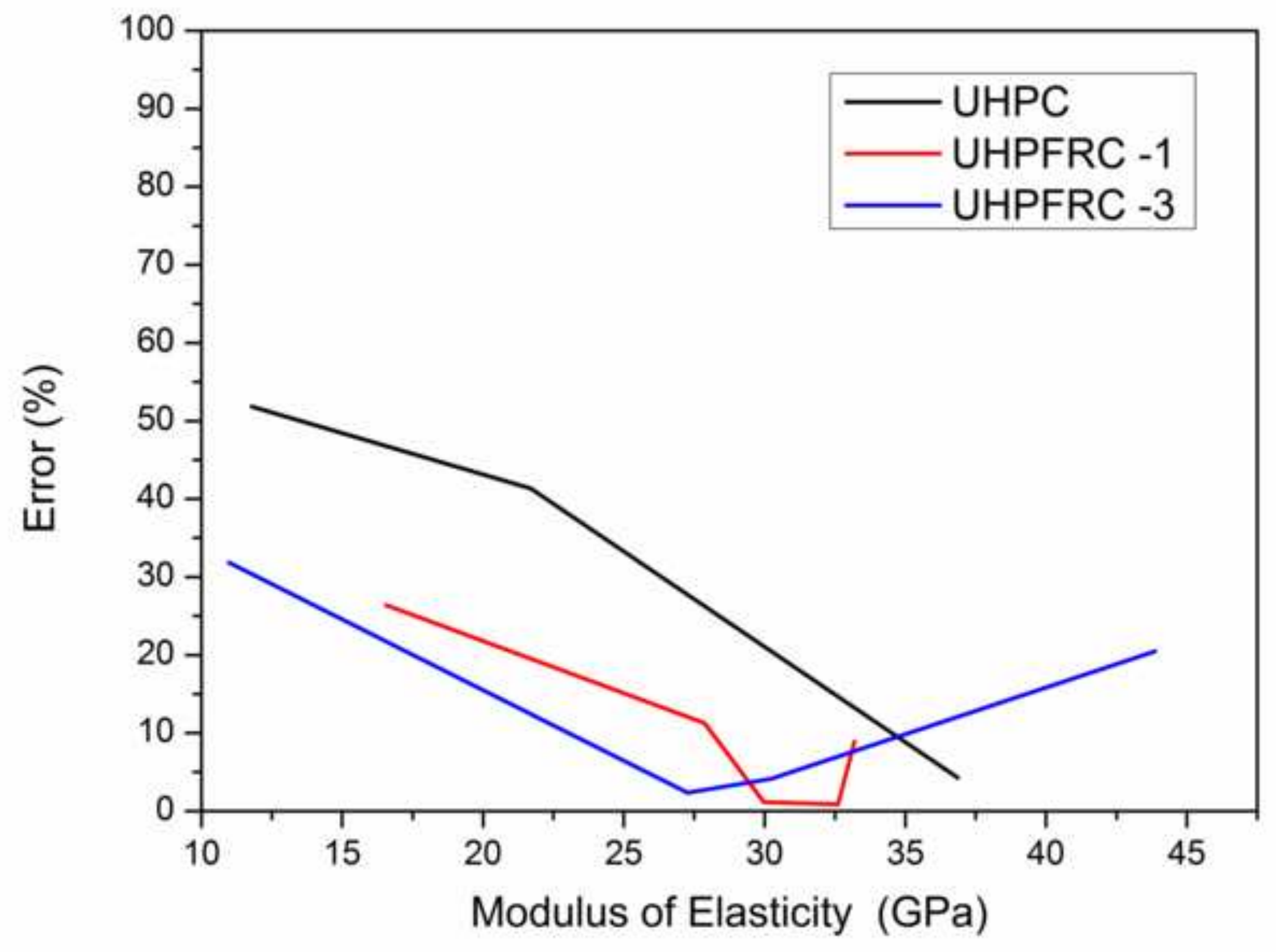




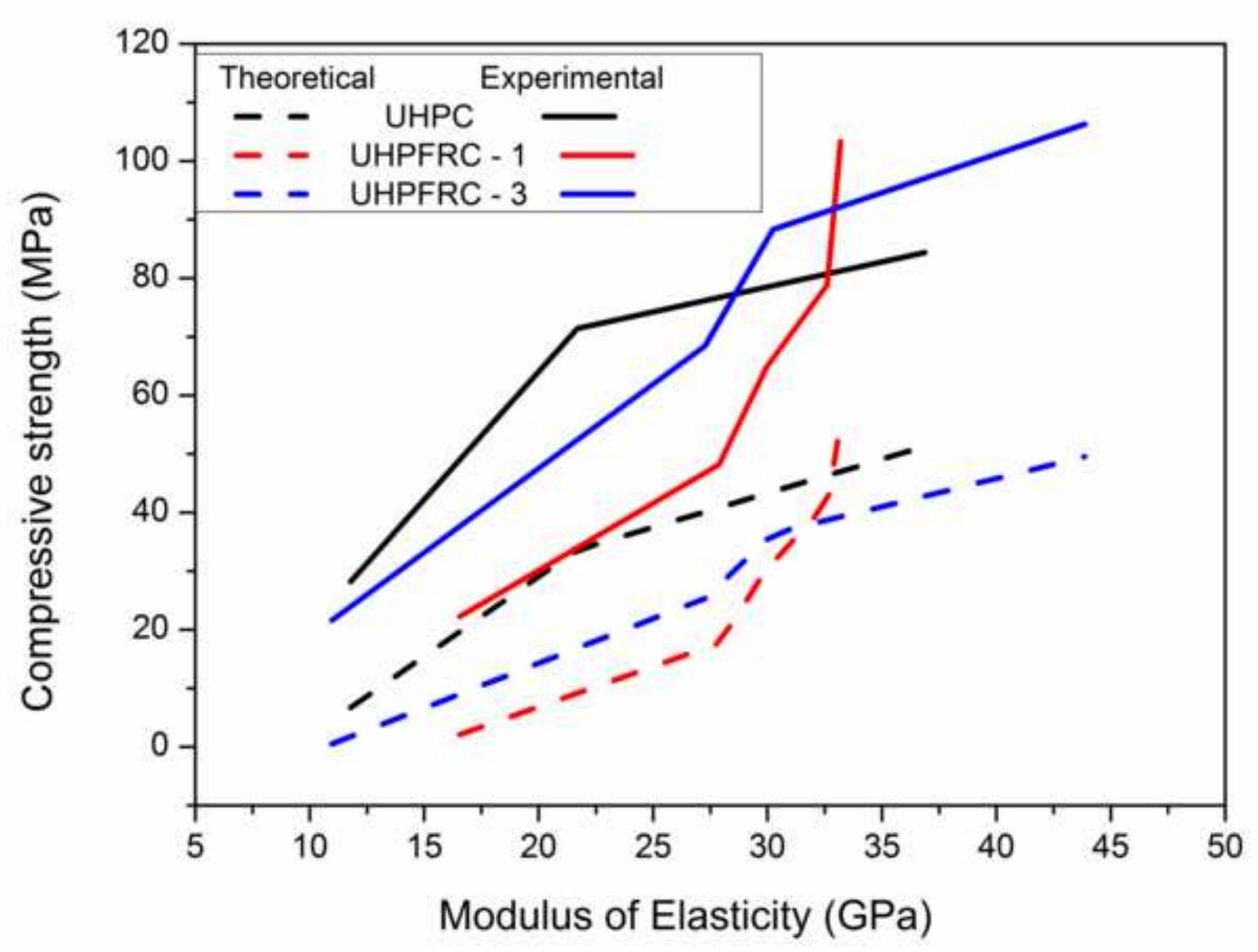




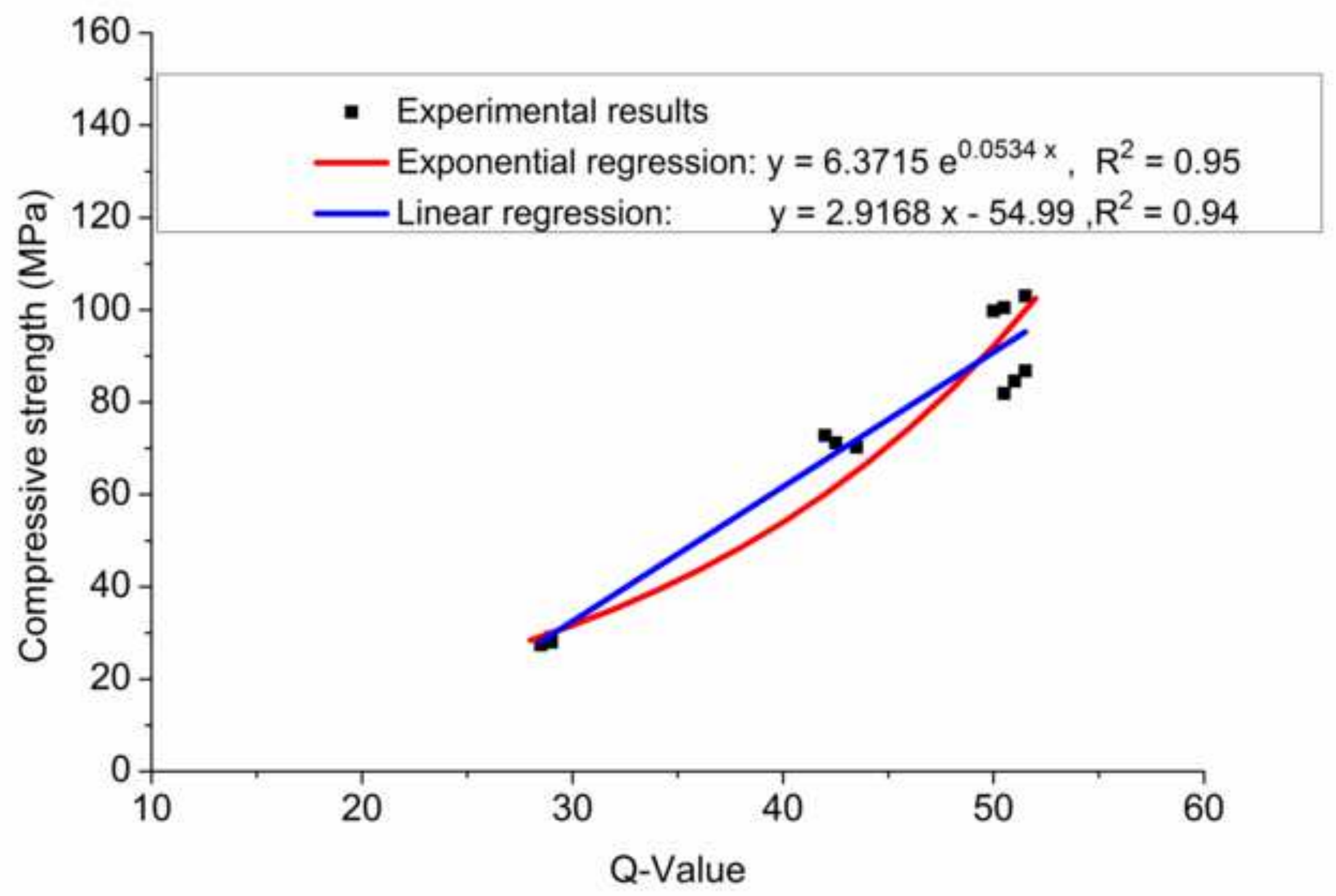




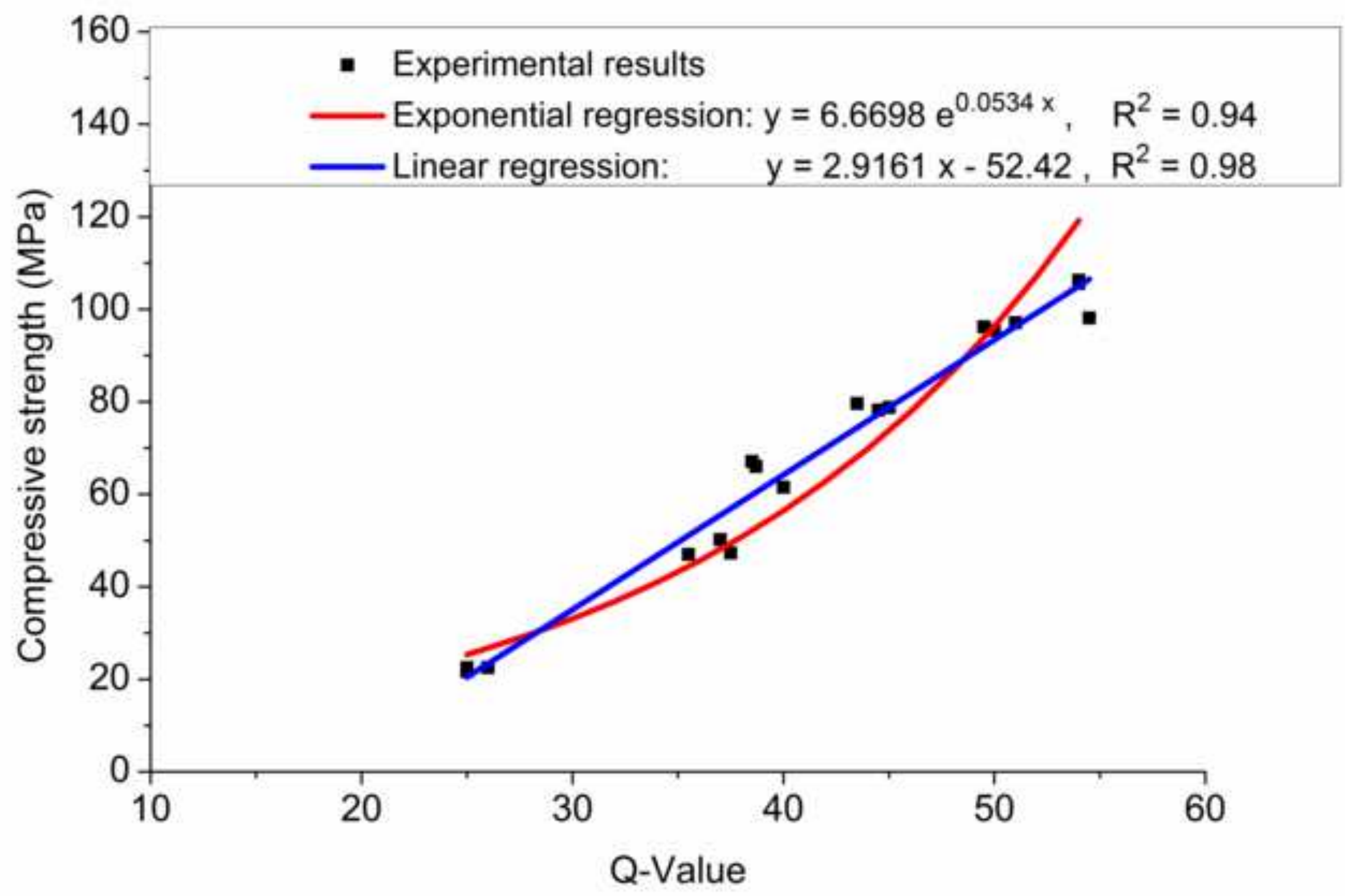




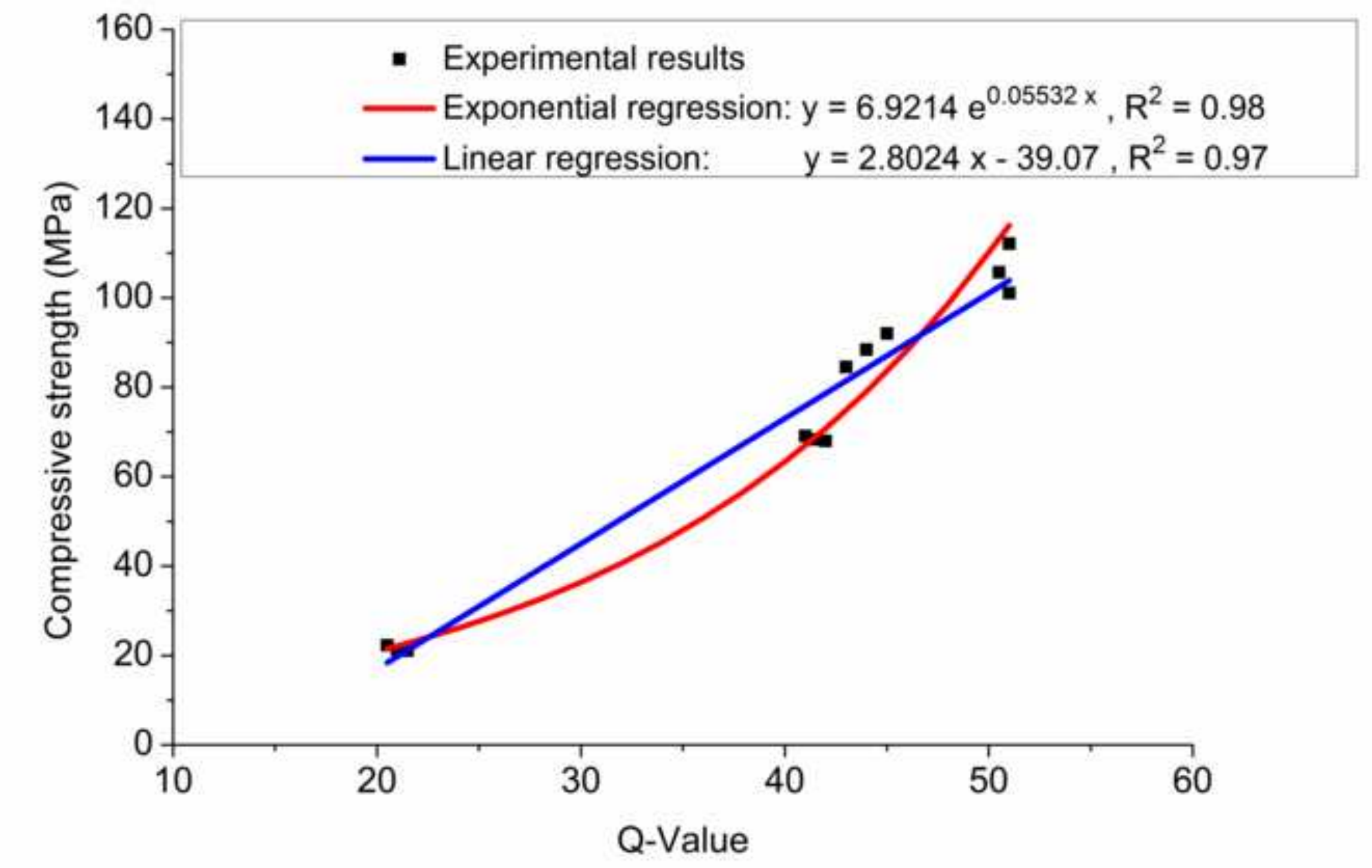




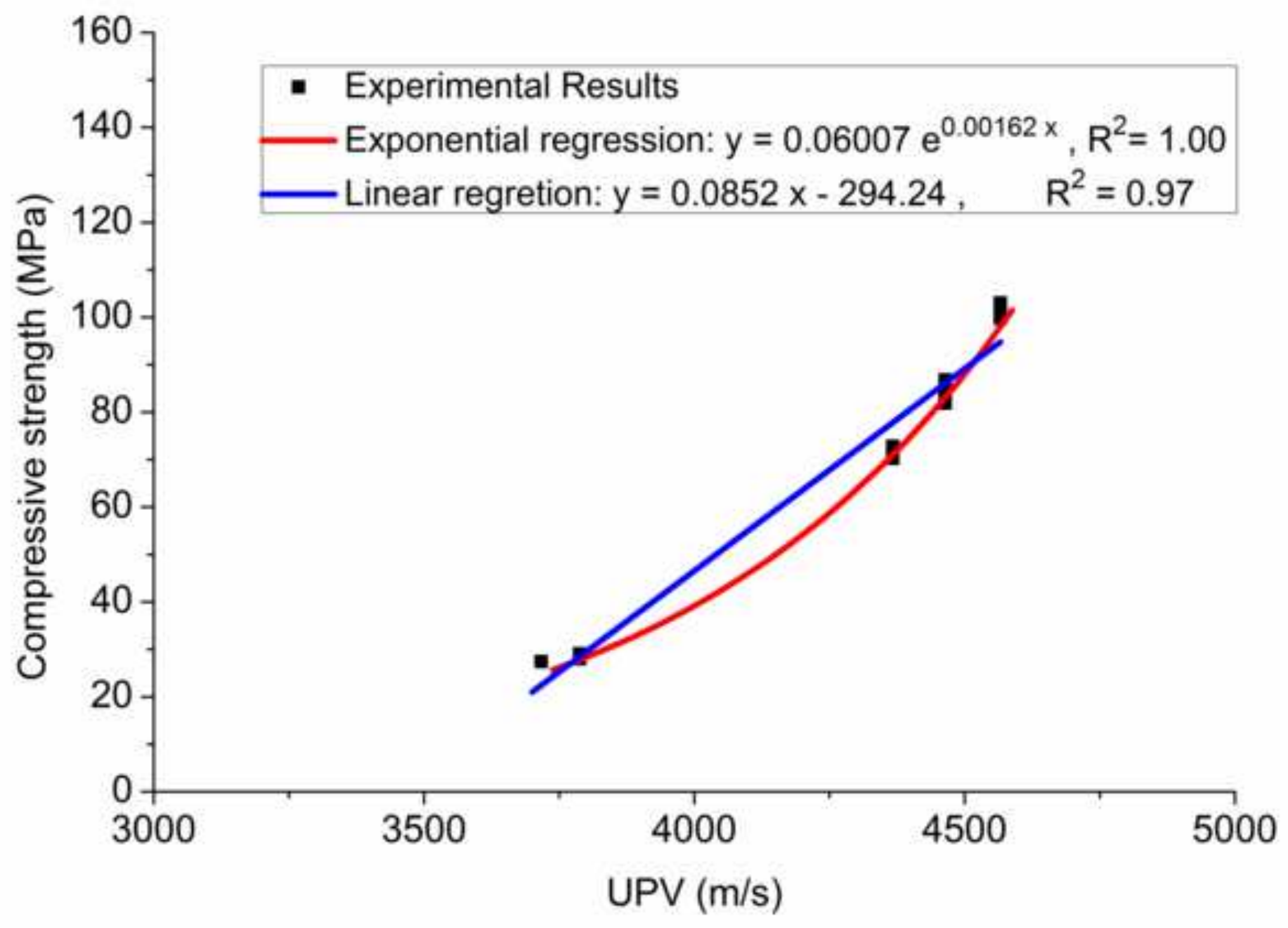




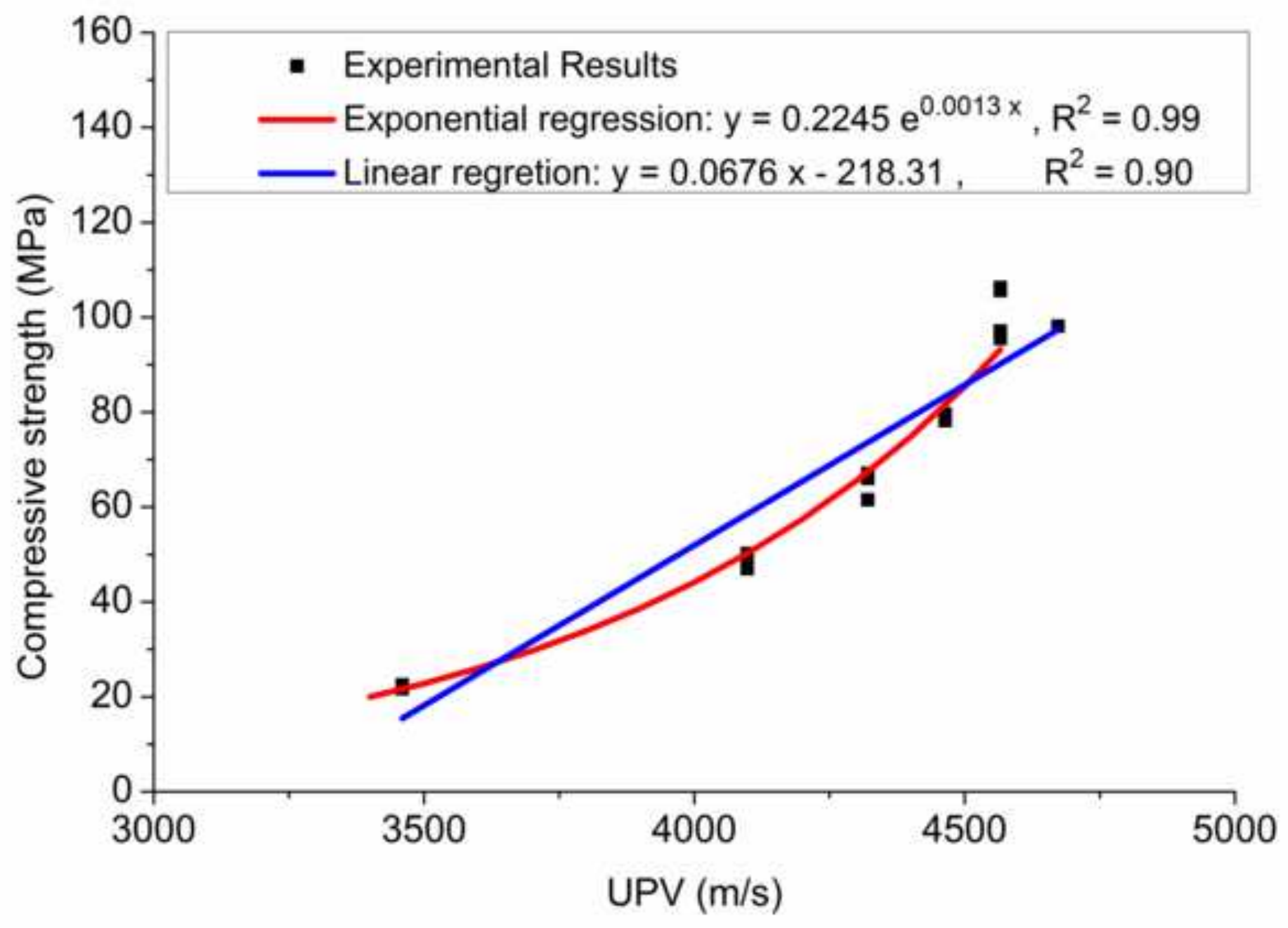




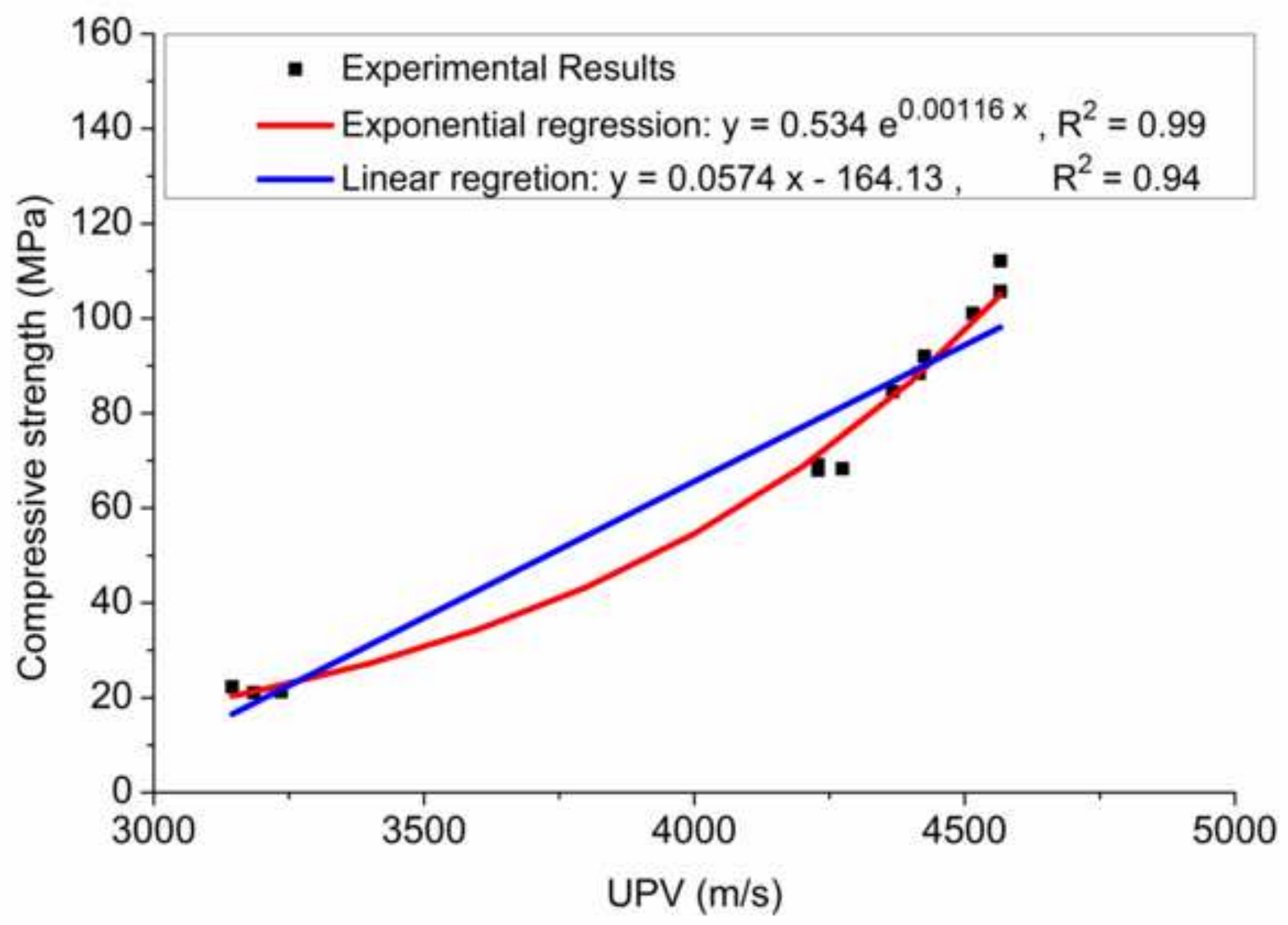




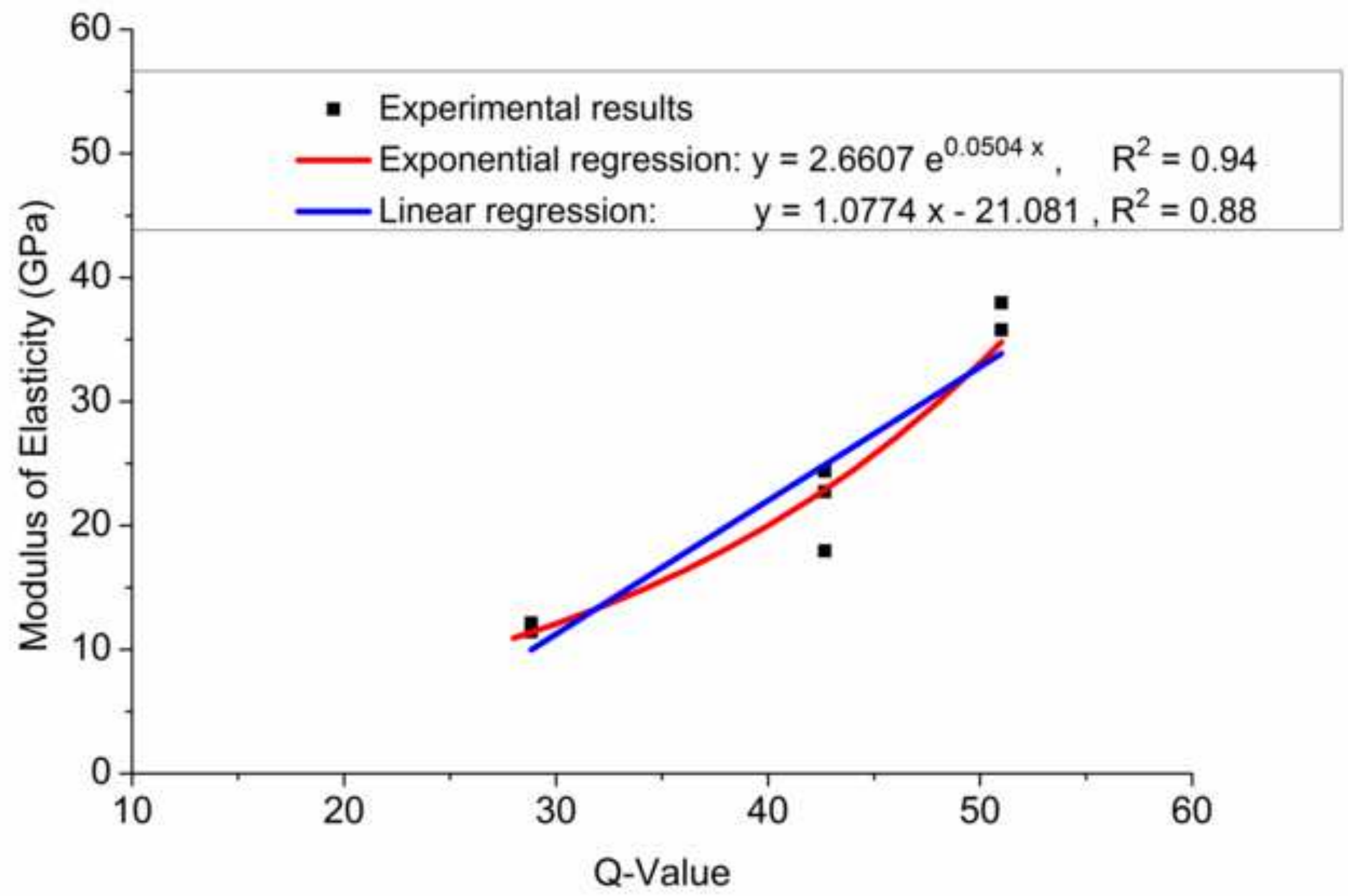




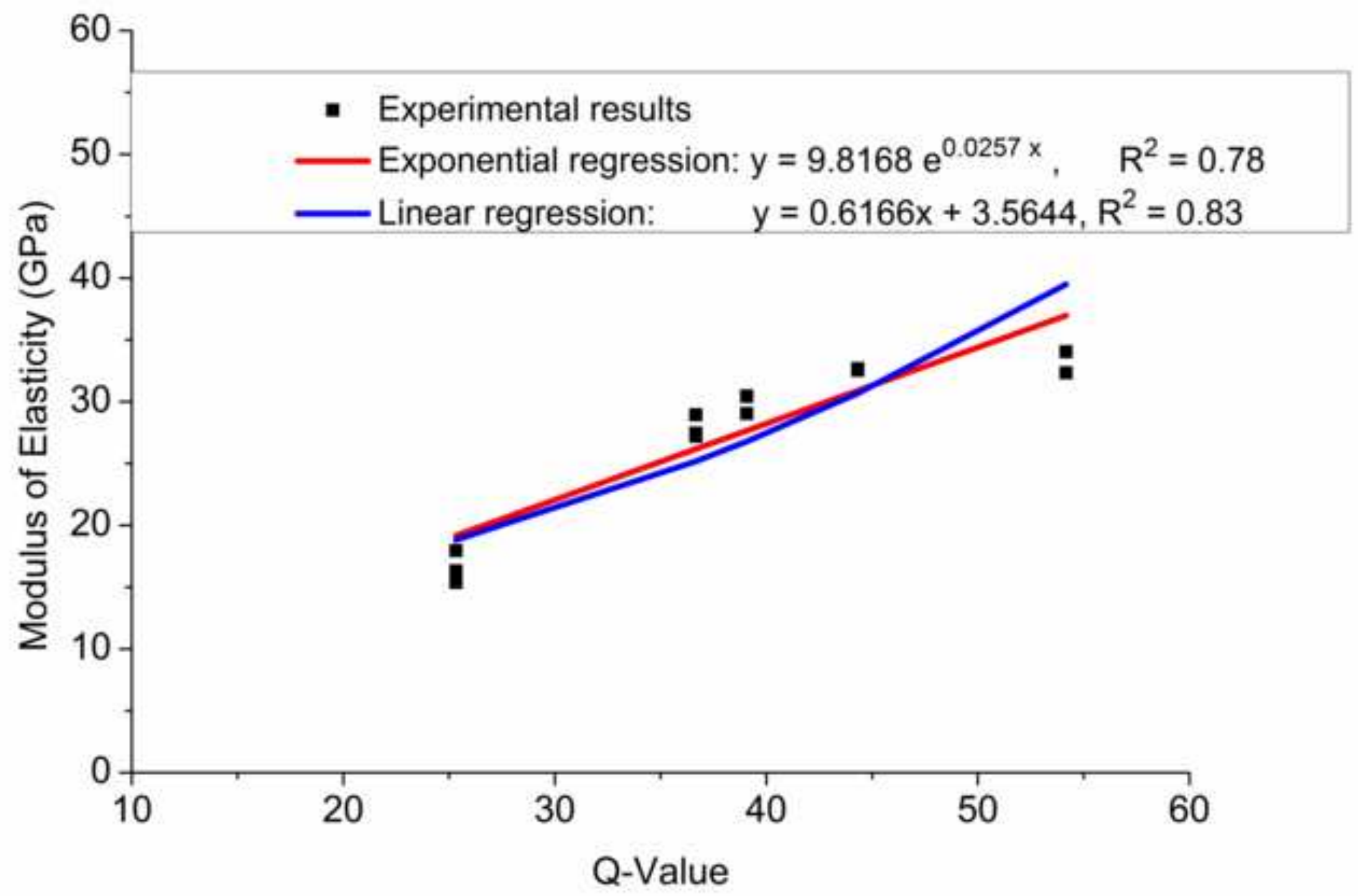




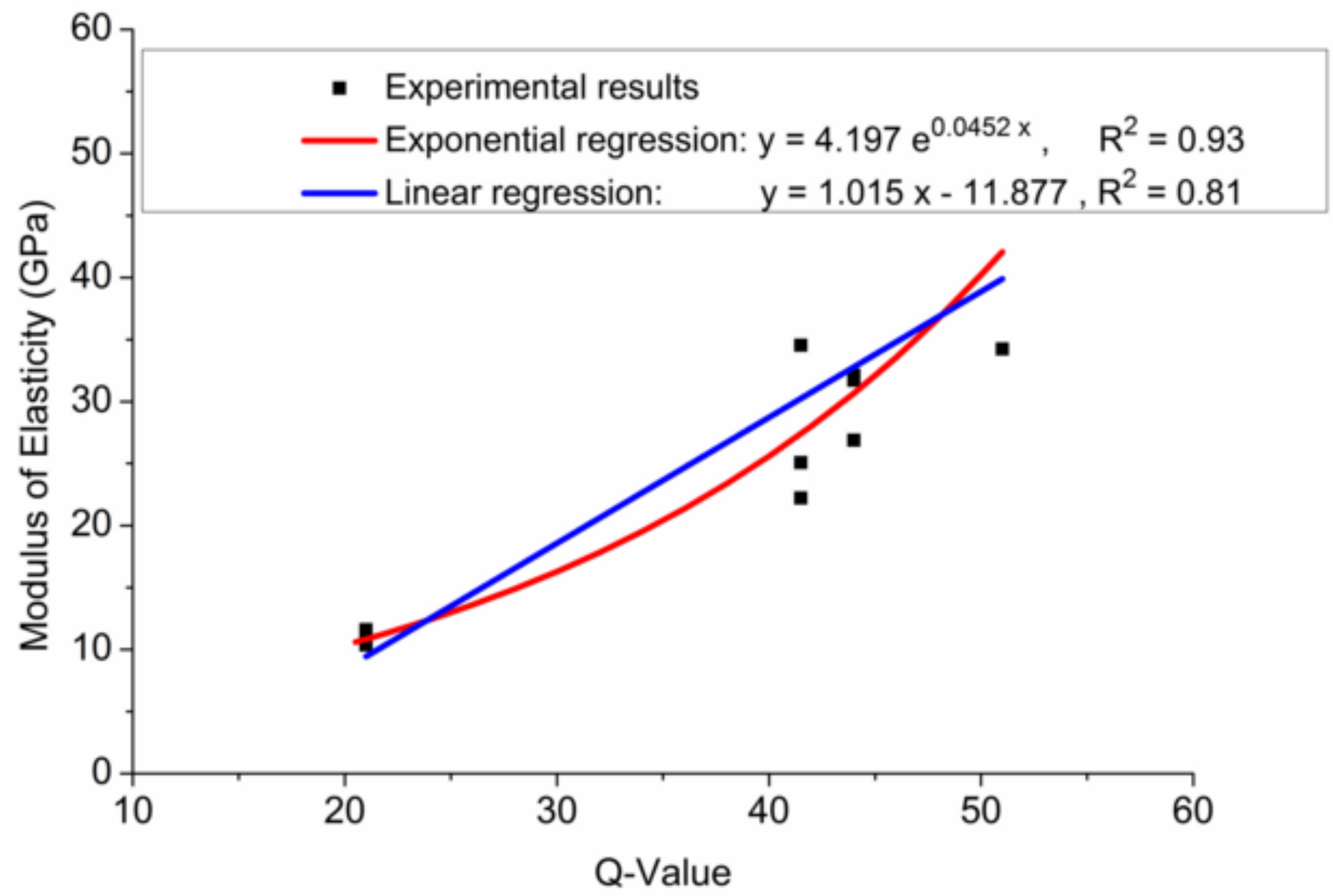




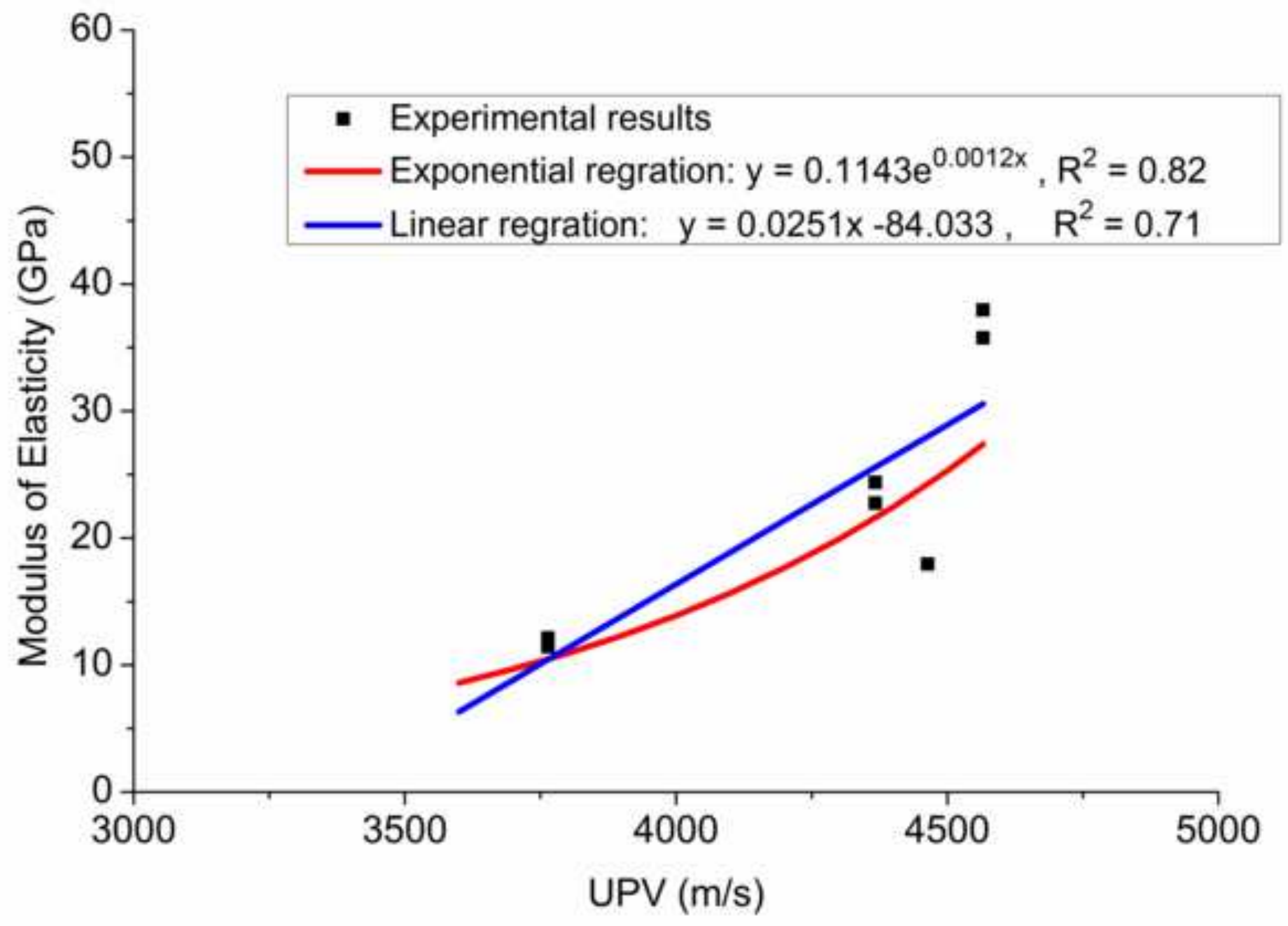




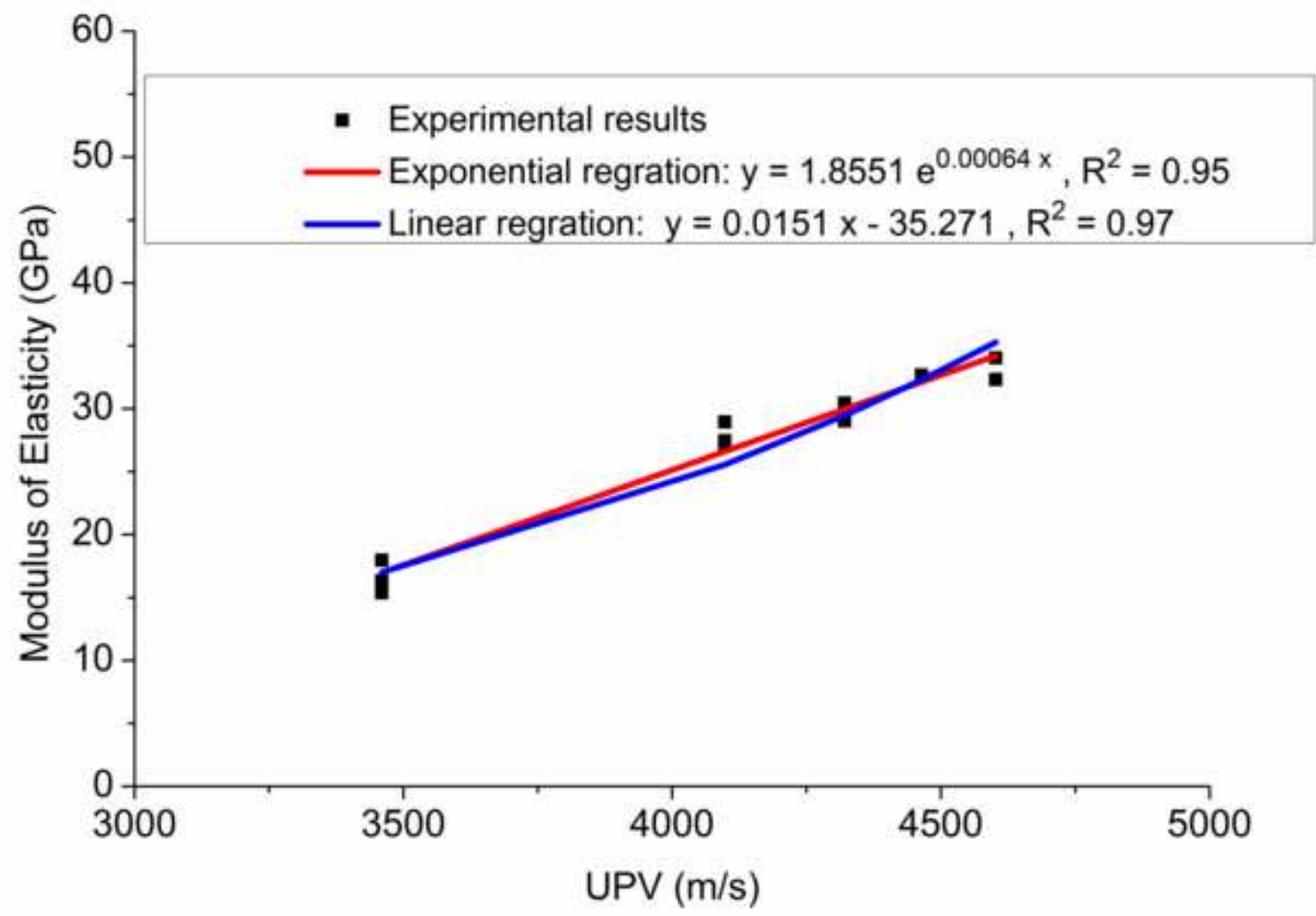




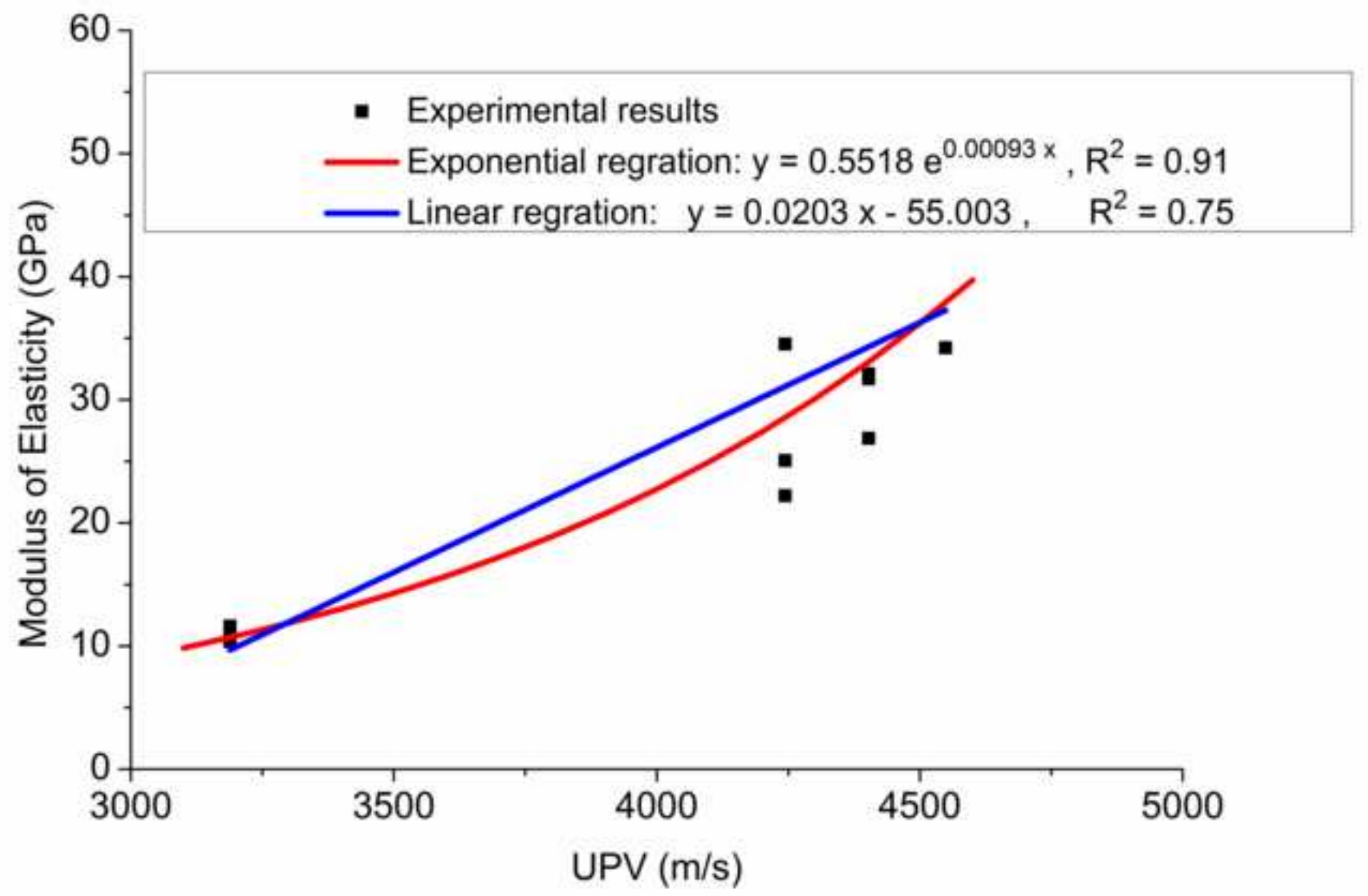




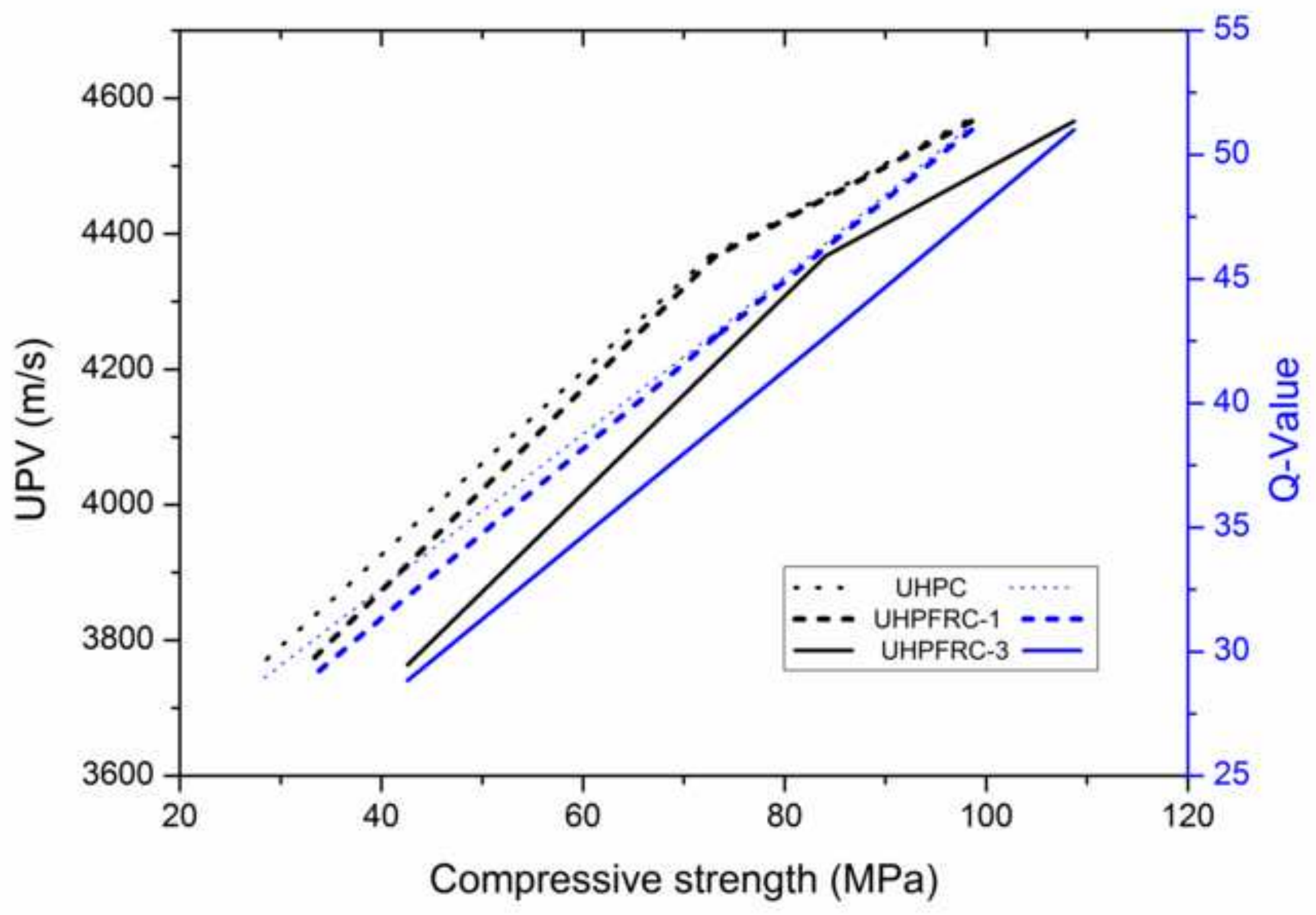




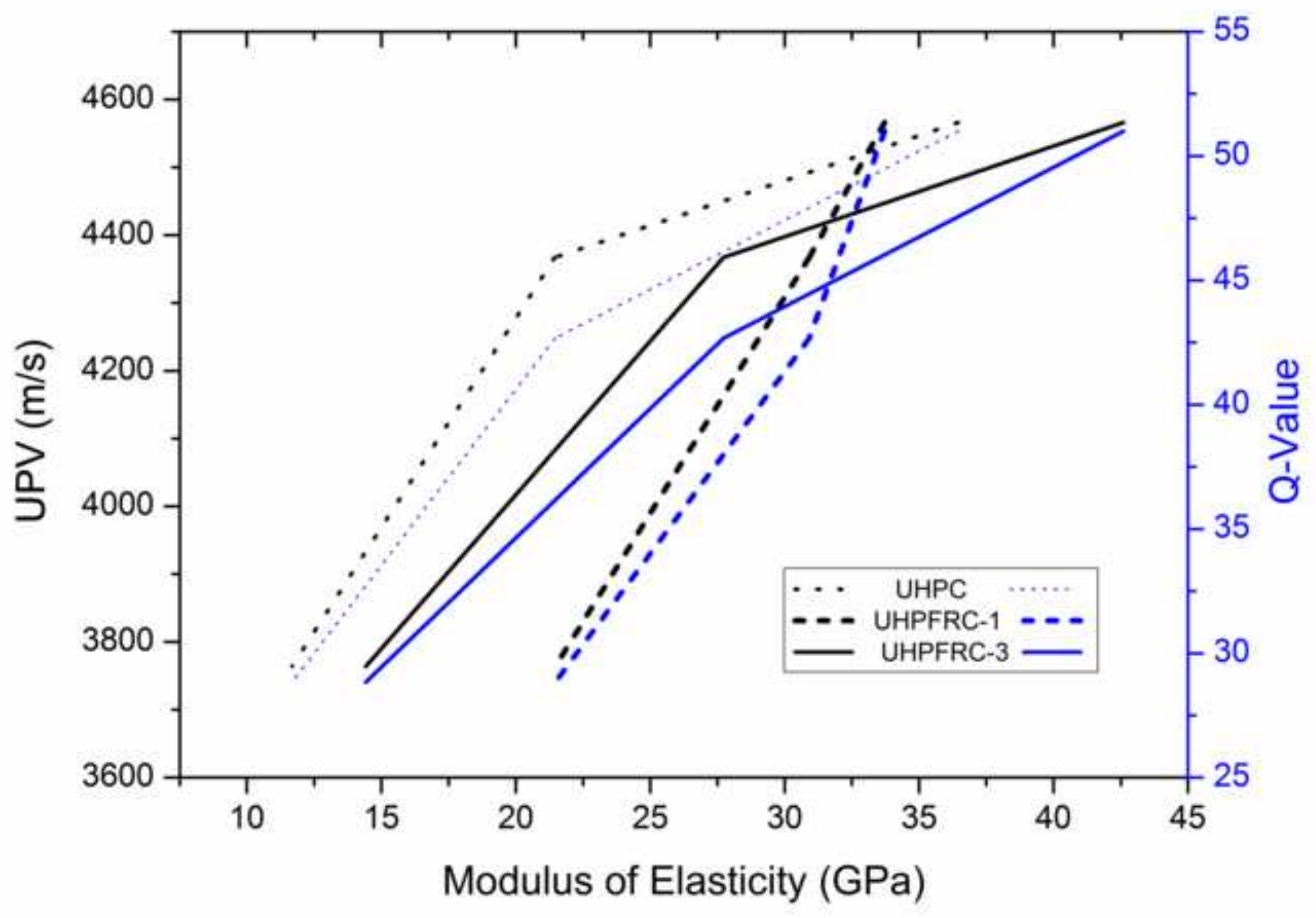




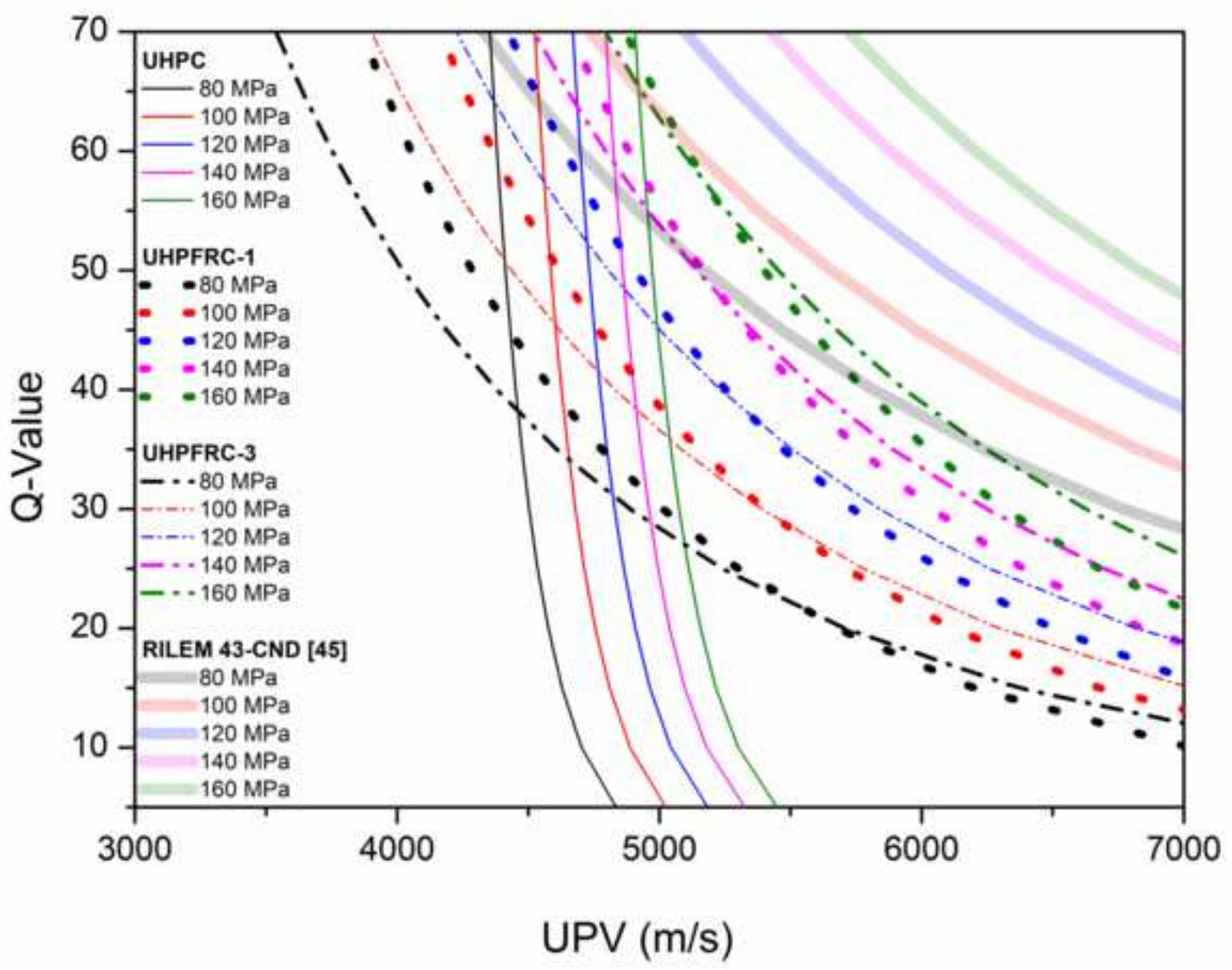




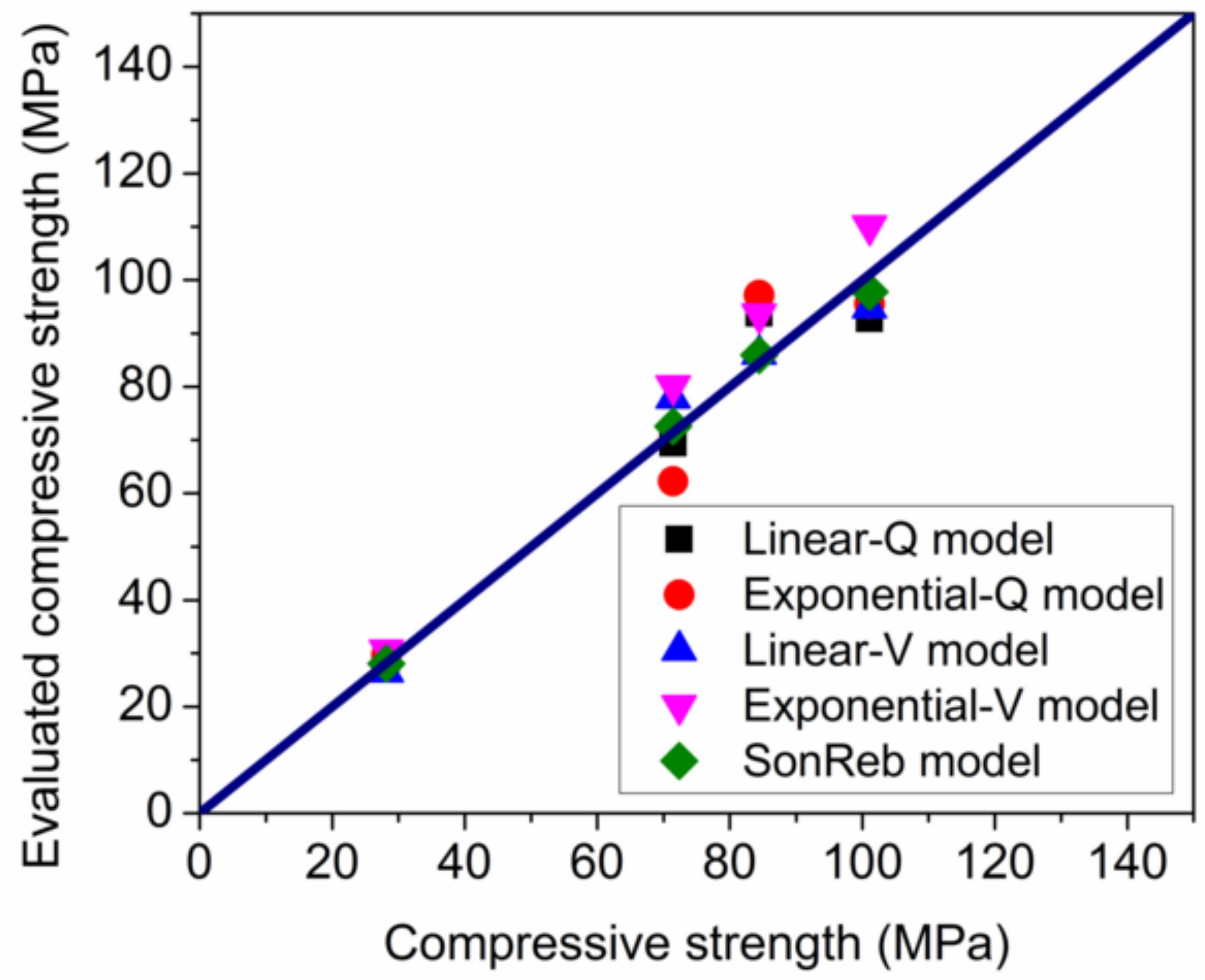




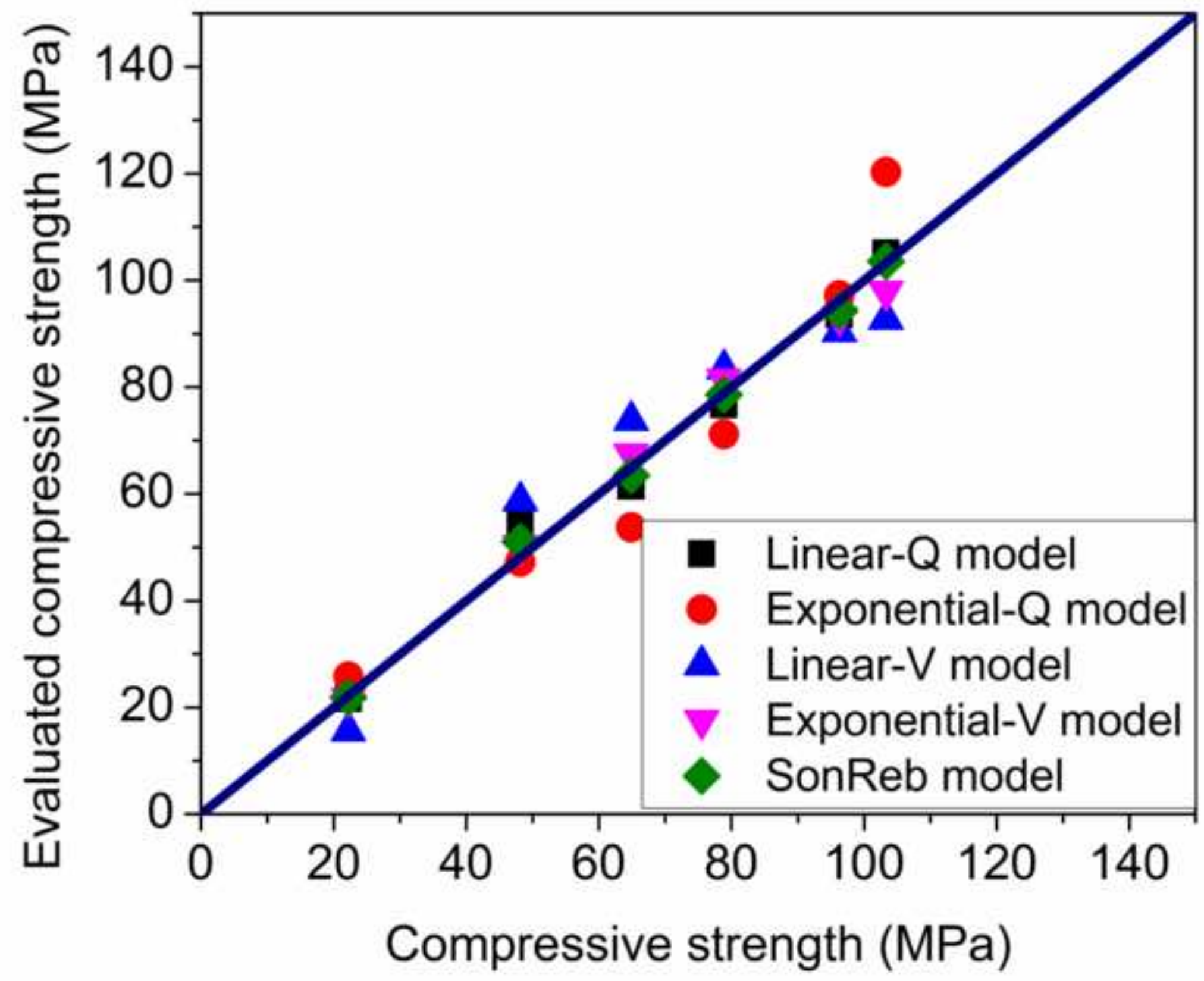




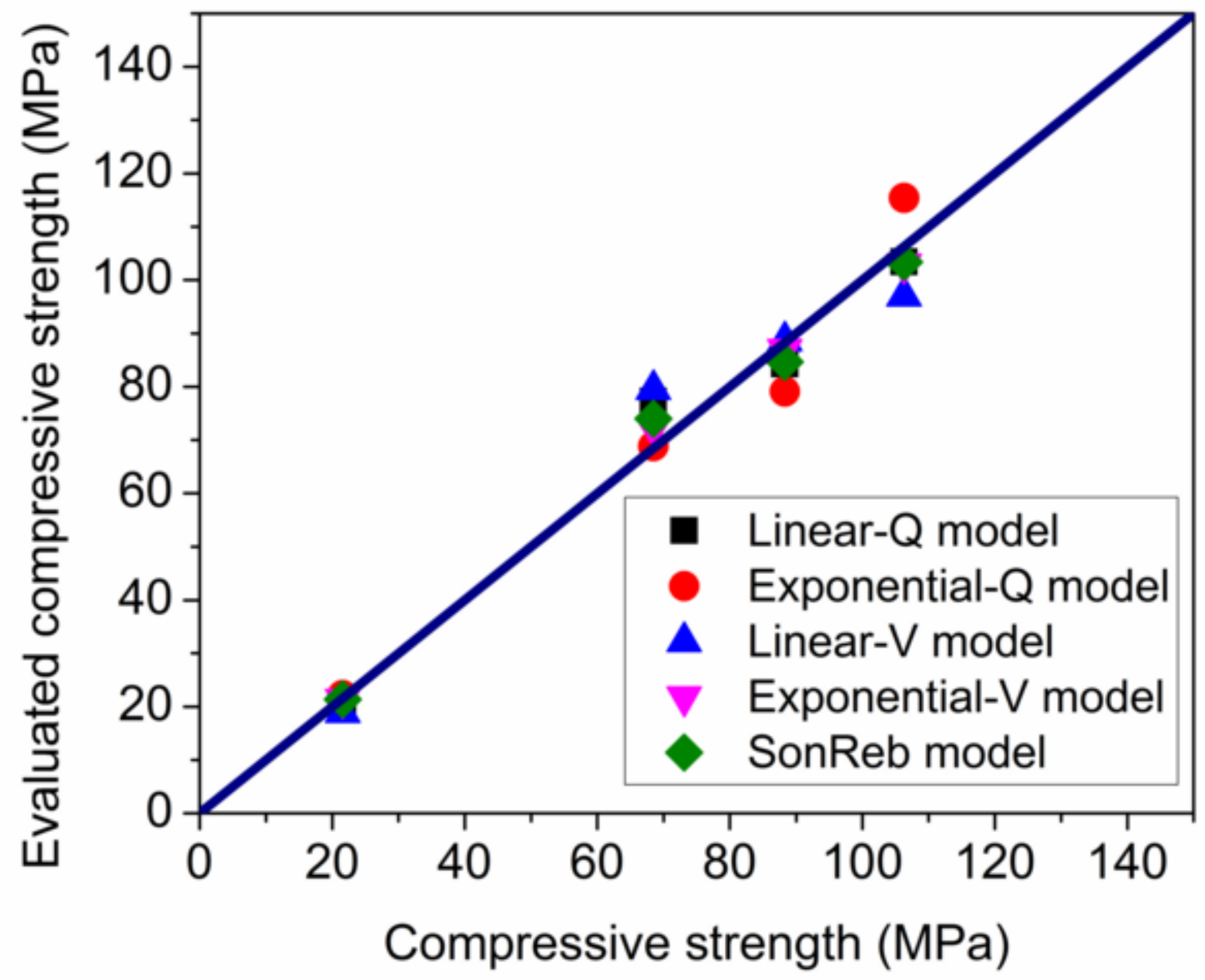




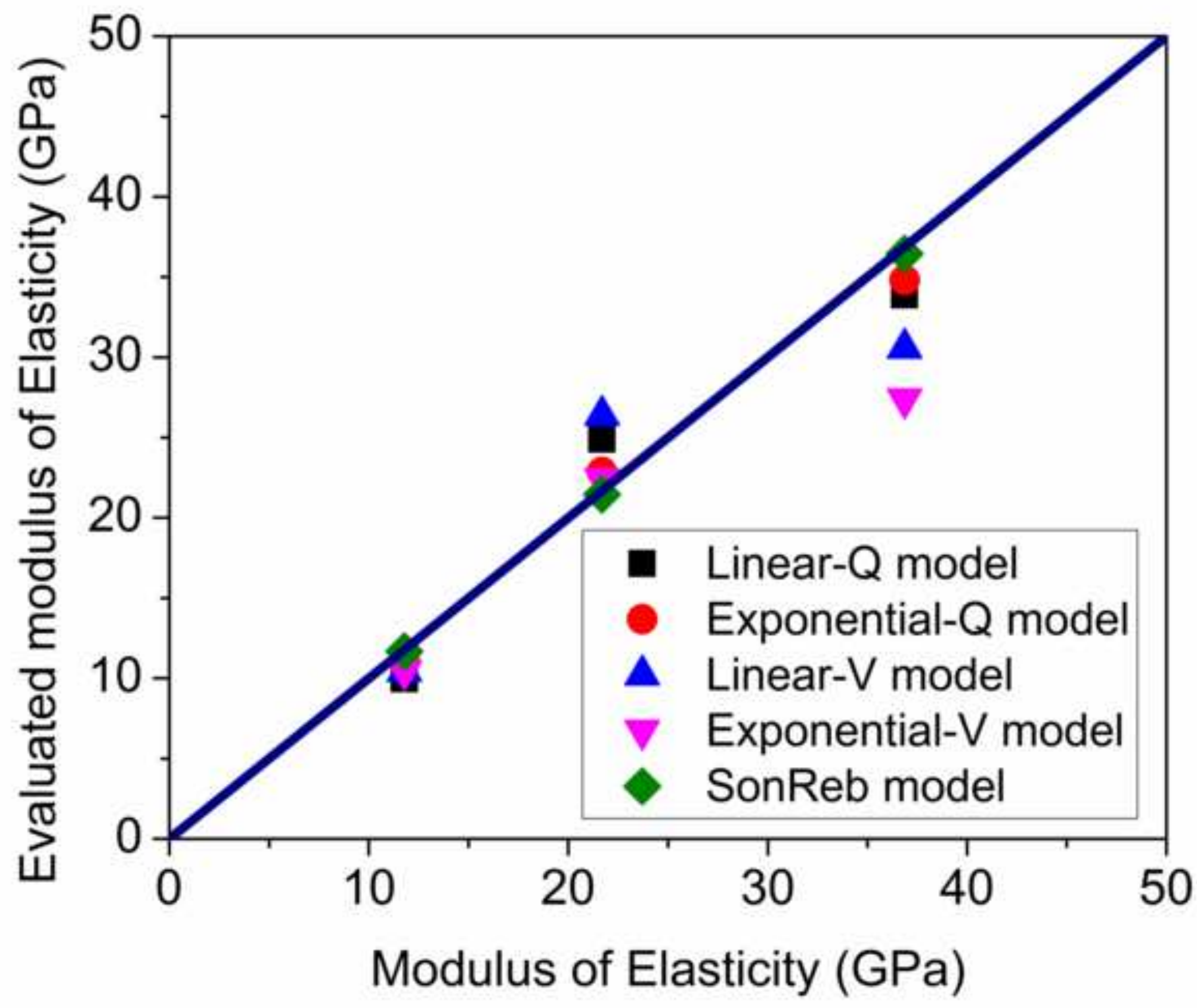




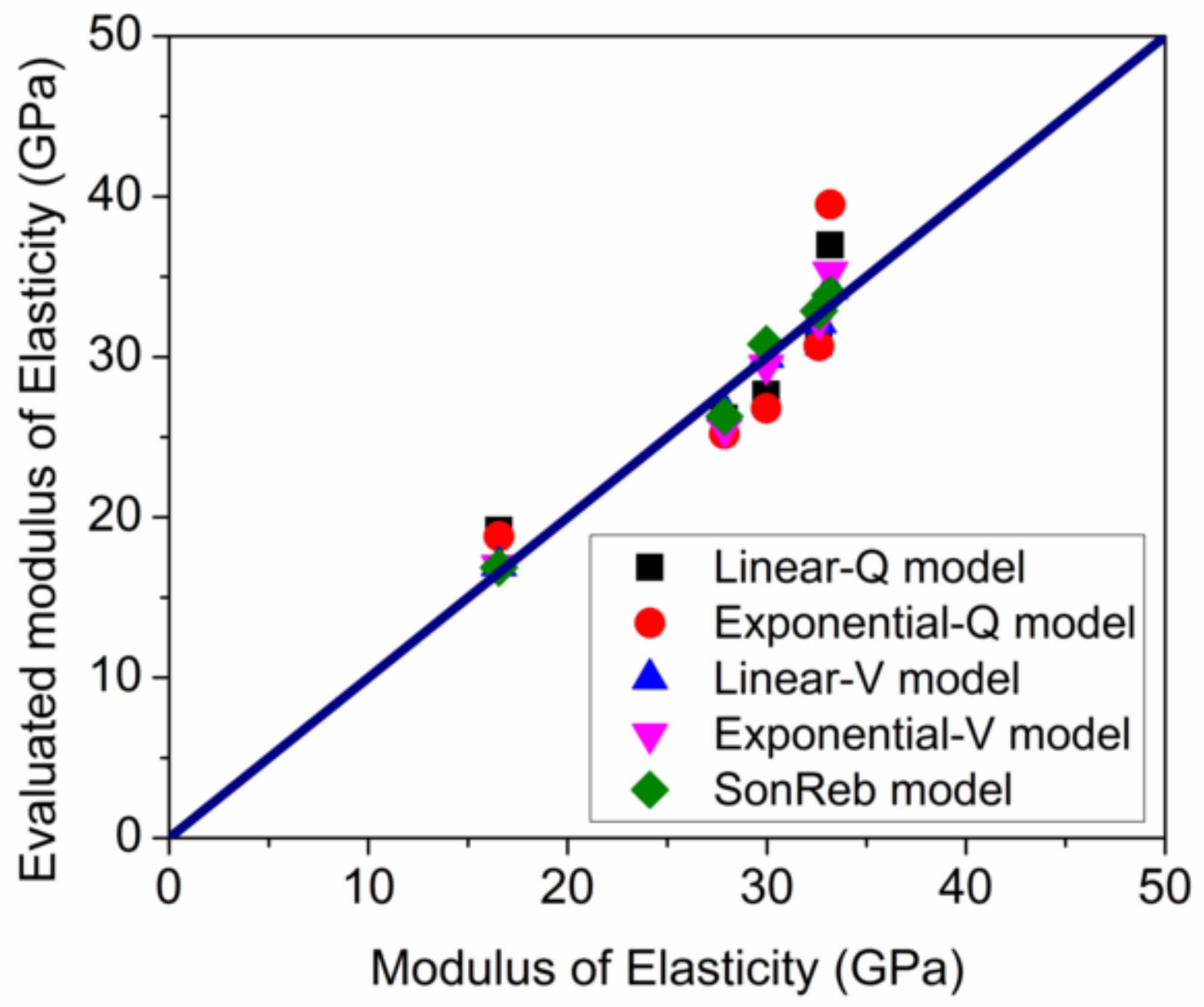




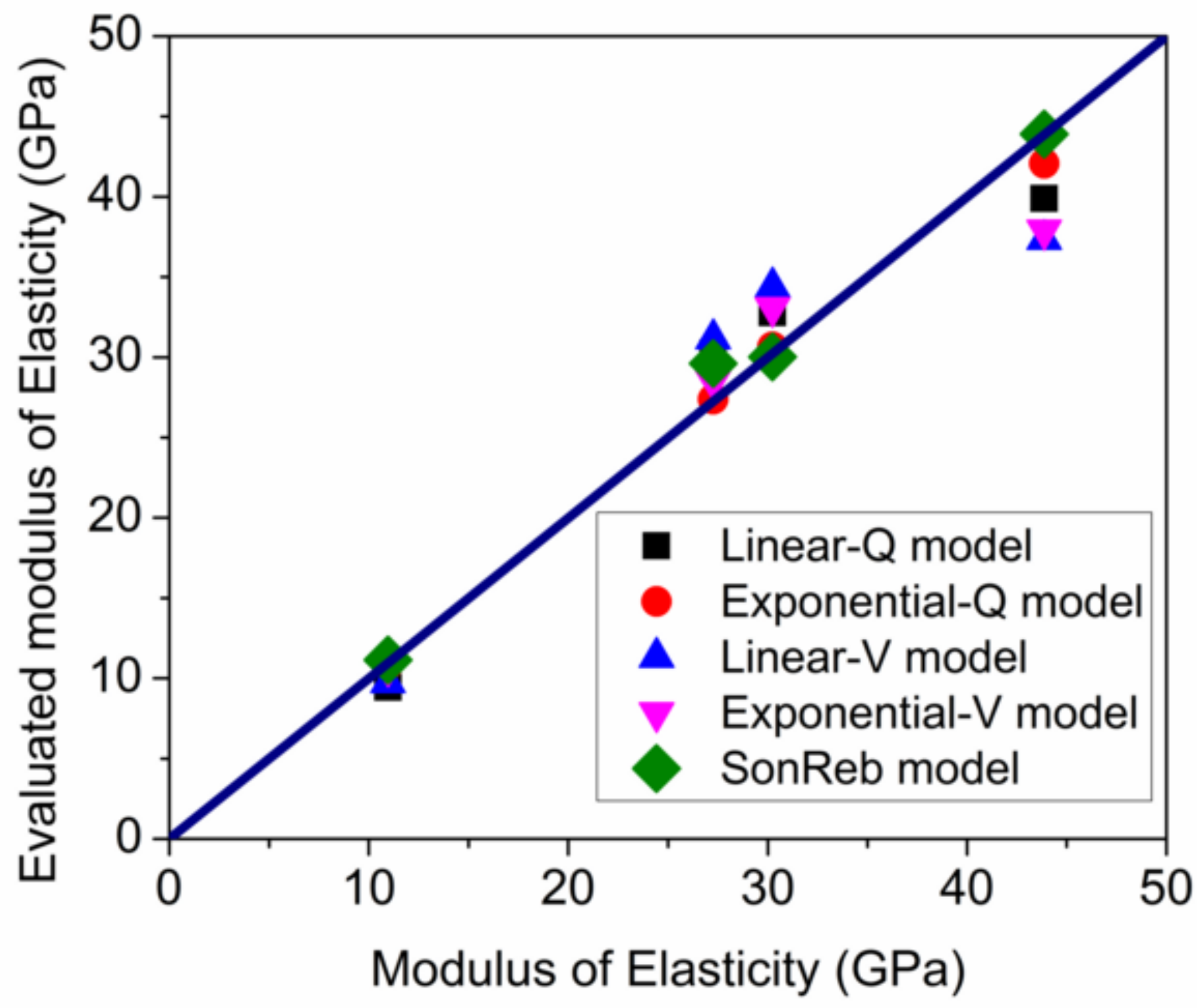




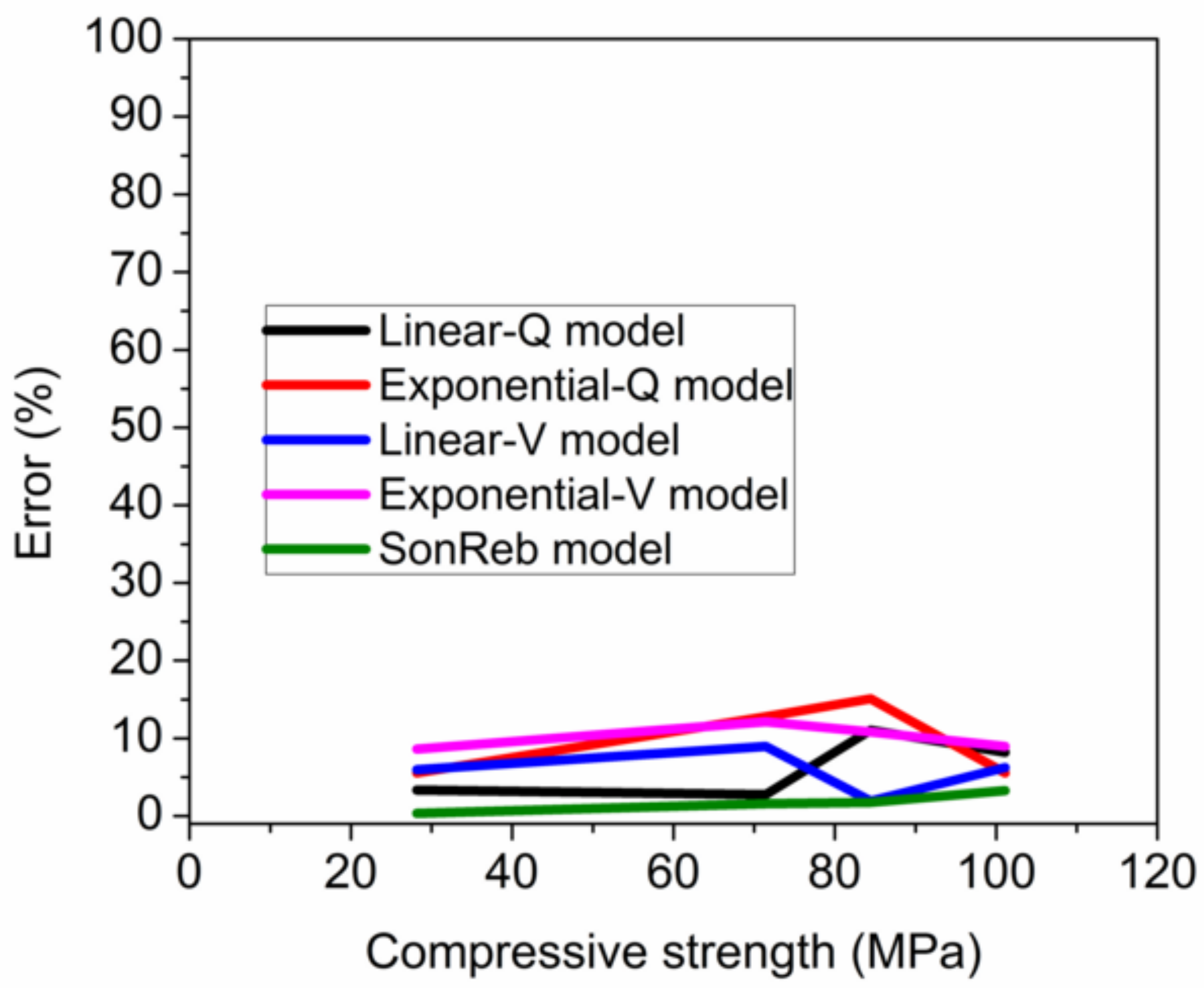




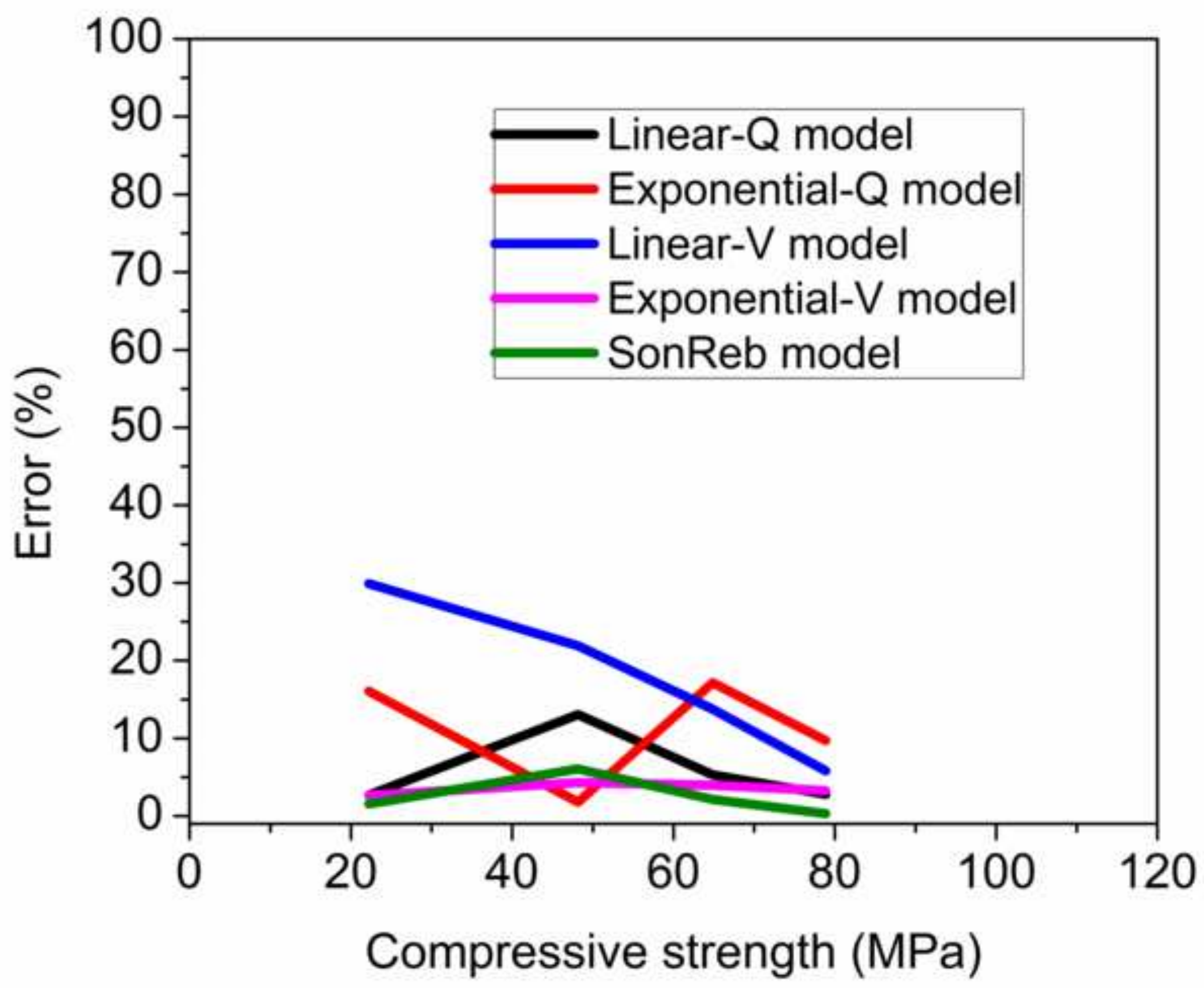




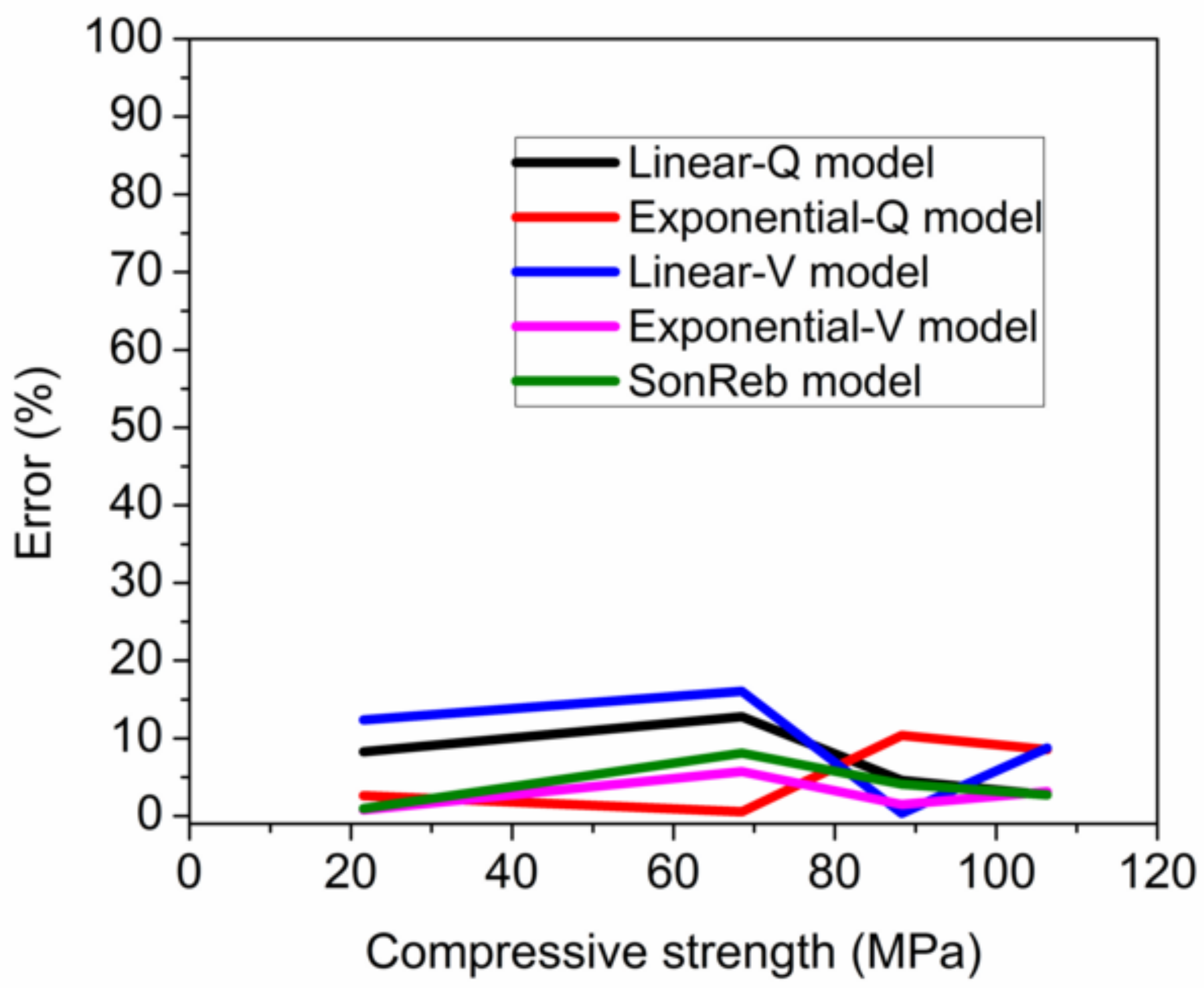




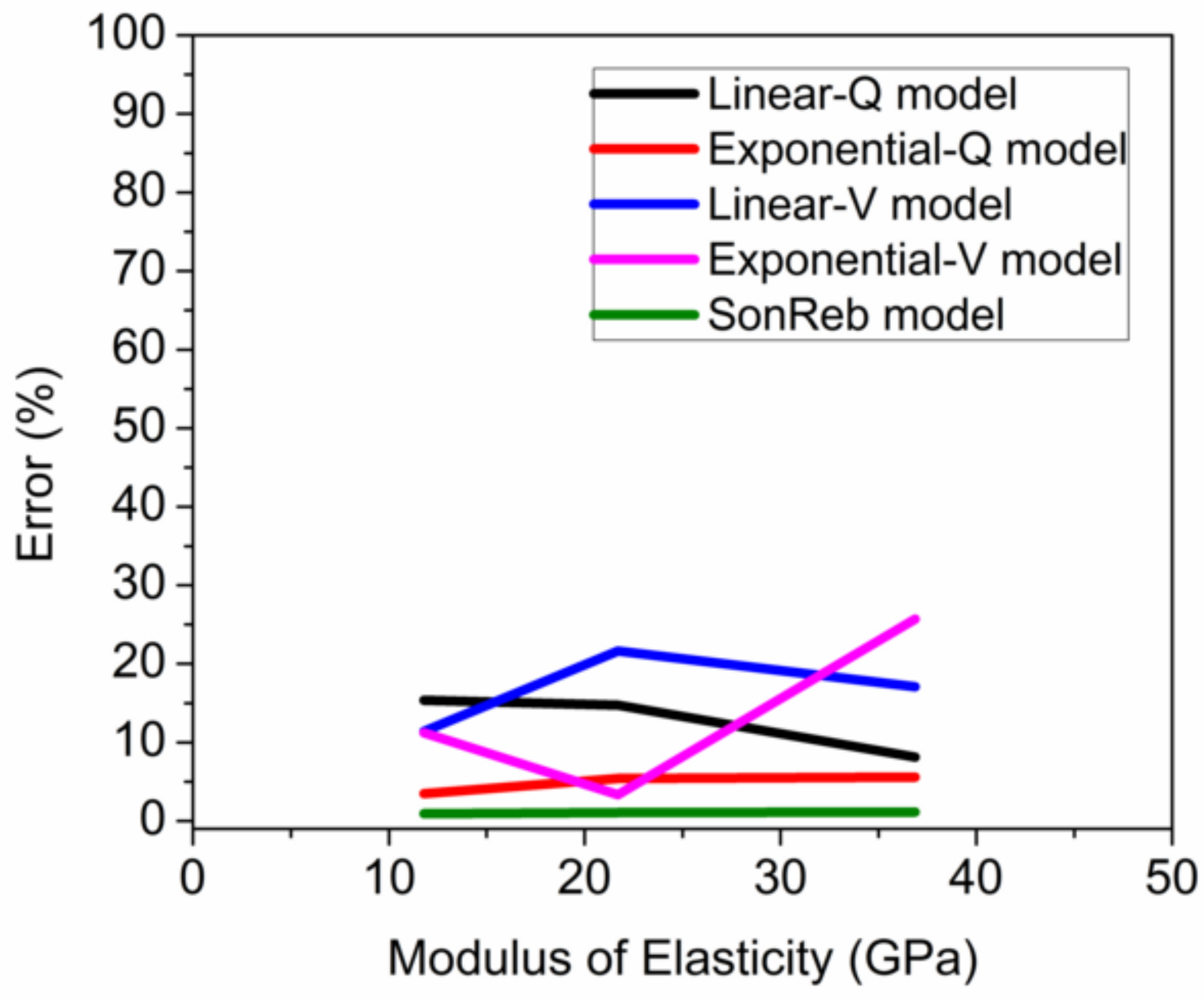




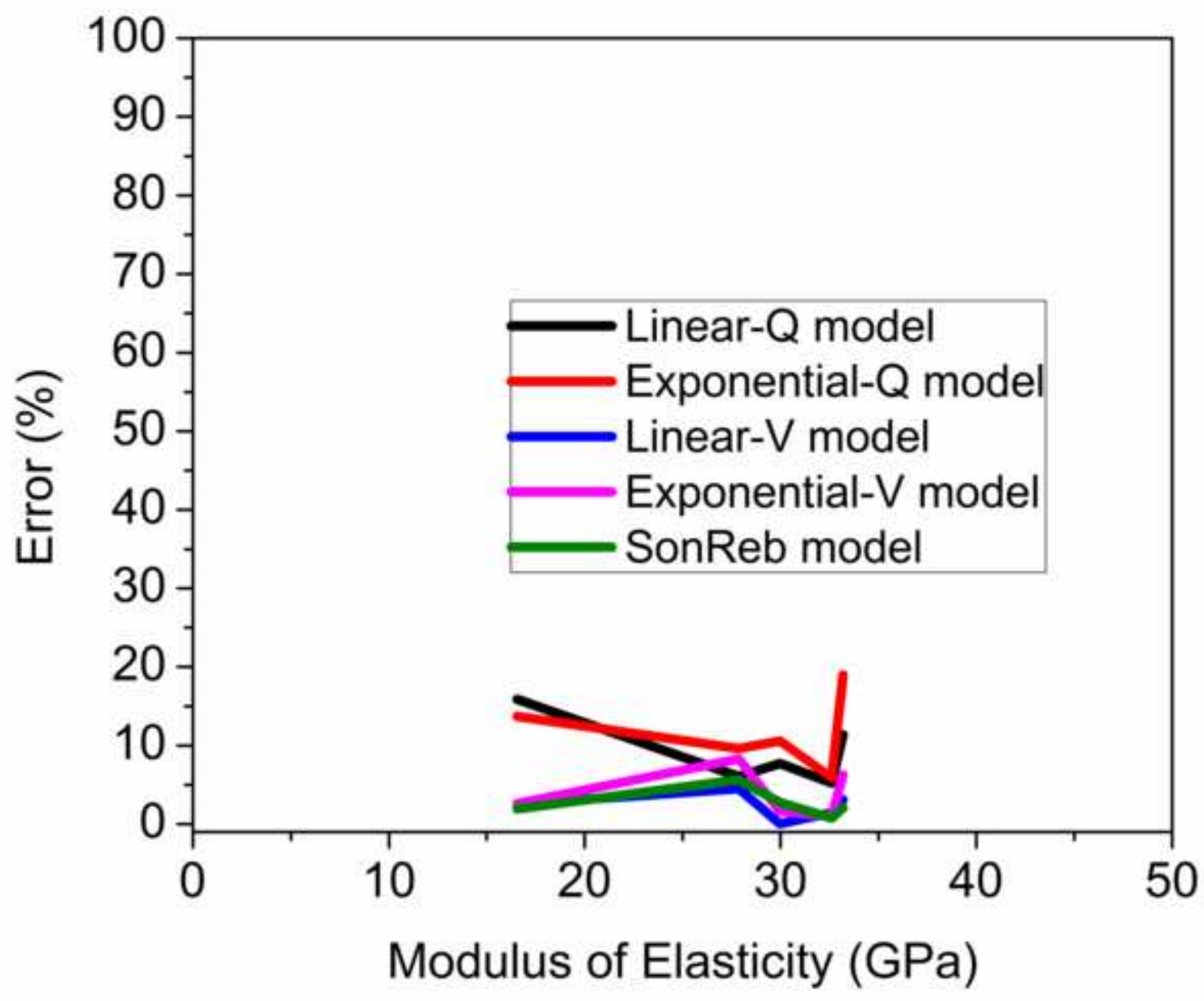




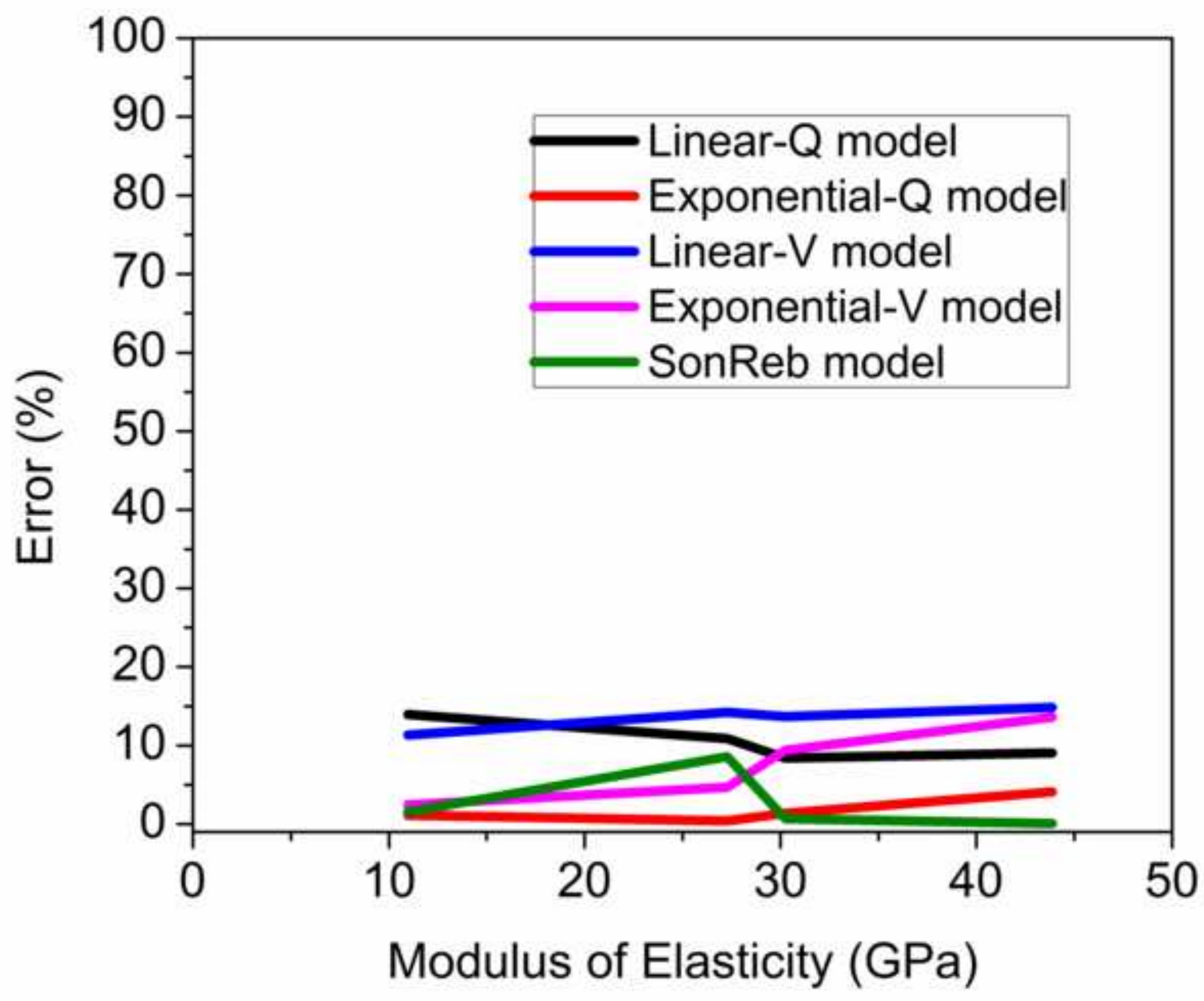

